HUTP-97/A014

CALT 68-2109

RU-97-24

\title{
Dual Descriptions of SO(10) SUSY Gauge Theories with Arbitrary Numbers of Spinors and Vectors
}

\author{
Micha Berkooz ${ }^{1}$, Peter $\mathrm{Cho}^{2}$, Per Kraus ${ }^{3}$ and Matthew J. Strassler ${ }^{2,4}$ \\ ${ }^{1}$ Department of Physics and Astronomy, Rutgers University, Piscataway, NJ 08855 \\ ${ }^{2}$ Lyman Laboratory, Harvard University, Cambridge, MA 02138 \\ ${ }^{3}$ Lauritsen Laboratory, California Institute of Technology, Pasadena, CA 91125 \\ ${ }^{4}$ School of Natural Sciences, Institute for Advanced Study, Princeton, NJ 08540
}

\begin{abstract}
We examine the low energy structure of $\mathcal{N}=1$ supersymmetric $S O(10)$ gauge theory with matter chiral superfields in $N_{Q}$ spinor and $N_{f}$ vector representations. We construct a dual to this model based upon an $S U\left(N_{f}+2 N_{Q}-7\right) \times S p\left(2 N_{Q}-2\right)$ gauge group without utilizing deconfinement methods. This product theory generalizes all previously known Pouliot-type duals to $S O\left(N_{c}\right)$ models with spinor and vector matter. It also yields large numbers of new dual pairs along various flat directions. The dual description of the $S O(10)$ theory satisfies multiple consistency checks including an intricate renormalization group flow analysis which links it with Seiberg's duality transformations. We discuss its implications for building grand unified theories that contain all Standard Model fields as composite degrees of freedom.
\end{abstract}




\section{Introduction}

During the past two years, significant theoretical interest and effort has been directed towards finding dual descriptions of strongly interacting $\mathcal{N}=1$ supersymmetric gauge theories. This enterprise was launched by Seiberg's construction of a dual to SUSY QCD [1]. Seiberg's discovery provided valuable insight into such general nonperturbative aspects of quantum field theory as confinement, massless solitons, phase transitions and conformal fixed points. A number of duality transformations uncovered since Seiberg's pioneering work have shed light upon other interesting phenomena including strong interaction cloaking of chirality [2] and dynamical supersymmetry breaking [3-9]. Unfortunately, no systematic field theory method for mapping long distance universality classes of microscopic supersymmetric gauge theories has so far been developed, and finding duals to models with more than just fundamental matter contents remains difficult. 1 A few specialized strategies which simplify the search have been devised by various groups. But given that the number of such theoretical tools is still quite limited, it is clearly worthwhile to discover and study more novel examples of duality.

In this article, we present an entire class of new dual pairs that exhibit several interesting patterns. One member of each pair consists of an $S O(10)$ gauge theory with matter in $N_{Q}$ spinor representations and $N_{f}$ vector representations. Its dual counterpart is a chiral model with semisimple gauge group $S U\left(N_{f}+2 N_{Q}-7\right) \times S p\left(2 N_{Q}-2\right)$. 2 Duals involving product groups have appeared before in the literature [4 0,20 23]. Many of these dualities can be derived by straightforward application of Seiberg's results [1] to individual group factors. Others [18,24,25] represent nontrivial generalizations of the duality transformations of Seiberg and of Kutasov [26], but still connect two theories of the same Cartan class. For example, in such models one finds that an $S U(N) \times S O\left(N^{\prime}\right)$ theory with appropriate matter is dual to a similar $S U(\tilde{N}) \times S O\left(\tilde{N}^{\prime}\right)$ theory. The product duals which we analyze in this paper qualitatively differ from these earlier examples. Moreover, they generalize all previously known Pouliot-type duals to various $S O\left(N_{c}\right)$ gauge theories with particular spinor and vector matter contents [2, 3,27 [30]. As we shall see, this new double array of dual pairs provides several novel insights into $\mathcal{N}=1$ duality.

1 Recent D-brane work of several investigators has led to a deeper understanding of $\mathcal{N}=1$ duality [10 19]. In this article, we follow a field theory approach to the subject.

2 We take the fundamental irrep of $S p(2 N)$ to be $2 N$ dimensional. 
Our article is organized as follows. We first focus upon the $S O(10)$ theory with two spinors and discuss its confining phase in section 2. We then construct the $S U\left(N_{f}-3\right) \times$ $S p(2)$ dual to the $N_{Q}=2$ theory in section 3 and verify that it satisfies multiple consistency checks including anomaly matching, composite operator mapping, and duality preservation along flat directions. In section 4 which contains our main results, we investigate the general product duals to $S O(10)$ models with arbitrary numbers of spinor and vector matter fields. We use these duals in section 5 to build grand unified theories that contain all Standard Model bosons and fermions as composites. Finally, we summarize our findings in section 6 and present details on exotic operator maps, two corollary duality transformations and an intricate renormalization group flow analysis in four separate appendices.

\section{The $N_{Q}=2 S O(10)$ model}

We begin our study by considering a supersymmetric gauge theory with symmetry group

$$
G=S O(10)_{\text {local }} \times\left[S U\left(N_{f}\right) \times S U(2) \times U(1)_{Y} \times U(1)_{R}\right]_{\text {global }},
$$

chiral superfields

$$
\begin{aligned}
V_{\mu}^{i} & \sim\left(10 ; \square, 1 ;-4, \frac{N_{f}-4}{N_{f}+4}\right) \\
Q_{I}^{A} & \sim\left(16 ; 1,2 ; N_{f}, \frac{N_{f}-4}{N_{f}+4}\right)
\end{aligned}
$$

and zero tree level superpotential. The hypercharge and R-charge assignments for the vector and spinor matter fields are chosen so that the model is free of global anomalies. It is also asymptotically free so long as its Wilsonian beta function coefficient 3

$$
b_{0}=\frac{1}{2}\left[3 K(\mathrm{Adj})-\sum_{\substack{\text { matter } \\ \text { reps } \rho}} K(\rho)\right]=20-N_{f}
$$

is positive. The beta function that governs the running of the physical gauge coupling is then negative at weak coupling [31]. The theory's infrared dynamics are consequently nontrivial provided it contains $N_{f}<20$ vectors.

Generic expectation values for the matter fields break the $S O(10)$ gauge group according to the pattern [32]

$$
S O(10) \stackrel{2(16)}{\longrightarrow} G_{2} \stackrel{10}{\longrightarrow} S U(3) \stackrel{10}{\longrightarrow} S U(2) \stackrel{10}{\longrightarrow} 1 .
$$

3 We adopt the $S O(10)$ index values $K(10)=2, K(16)=4$, and $K(45)=16$. 
This expression illustrates the hierarchy of gauge symmetries realized at progressively longer distance scales, assuming that the spinor vevs' magnitudes are larger than the first vector's, which in turn is larger than the second vector's, and so forth. Using this symmetry breaking information, we can straightforwardly count the gauge invariant operators that are needed to act as moduli space coordinates for small numbers of vector flavors [2,33. Such gauge singlets enter into the effective low energy description of the microscopic $S O(10)$ theory. In Table 1, we display the number of partonic degrees of freedom and the generic unbroken color subgroup $H_{\text {local }}$ as a function of $N_{f}$. The dimension of the coset space $G_{\text {local }} / H_{\text {local }}$ coincides with the number of matter fields eaten by the superHiggs mechanism. The number of remaining uneaten partons listed in the last column of the table equals the number of independent hadrons which label flat directions in the effective theory.

\begin{tabular}{|c|c|c|c|c|}
\hline$N_{f}$ & Parton DOF & Unbroken Subgroup & Eaten DOF & Hadrons \\
\hline 0 & 32 & $G_{2}$ & $45-14=31$ & 1 \\
1 & 42 & $S U(3)$ & $45-8=37$ & 5 \\
2 & 52 & $S U(2)$ & $45-3=42$ & 10 \\
3 & 62 & 1 & 45 & 17 \\
4 & 72 & 1 & 45 & 27 \\
5 & 82 & 1 & 45 & 37 \\
\hline
\end{tabular}

Table 1: Number of independent hadrons in the $S O(10)$ theory with two spinors

In order to explicitly construct the hadron fields, it is useful to recall the tensor decomposition of the $S O(10)$ spinor product

$$
16 \times 16=10_{S}+120_{A}+126_{S}=[1]_{S}+[3]_{A}+\widetilde{[5]_{S}}
$$

Here $[n]$ denotes an antisymmetric rank- $n$ tensor irrep, the "S" and "A" subscripts indicate symmetry and antisymmetry under spinor exchange and the tilde over the last term implies 
that the rank-5 irrep is complex self-dual [34]. We form symmetric and antisymmetric combinations of the two spinors using the Pauli matrices $\sigma_{\mathrm{x}} \sigma_{2}(\mathrm{x}=1,2,3)$ and $\sigma_{2}$ as Clebsch-Gordan coefficients. We next contract vector superfields into the bispinor pairs utilizing $S O(10)$ Gamma matrices $\Gamma_{\mu}$ and charge conjugation matrix $C$. $⿴$. We thus produce the gauge invariant composites

$$
\begin{aligned}
& K=Q_{I}^{T}\left(\sigma_{\mathrm{x}} \sigma_{2}\right)_{I J} \Gamma^{\mu} C Q_{J} Q_{K}^{T}\left(\sigma_{\mathrm{x}} \sigma_{2}\right)_{K L} \Gamma_{\mu} C Q_{L} \sim\left(1 ; 1,1 ; 4 N_{f}, 4 \frac{N_{f}-4}{N_{f}+4}\right) \\
& M^{(i j)}=\left(V^{T}\right)^{i \mu} V_{\mu}^{j} \sim\left(1 ; \square, 1 ;-8,2 \frac{N_{f}-4}{N_{f}+4}\right) \\
& N_{\mathrm{x}}^{i}=Q_{I}^{T}\left(\sigma_{\mathrm{x}} \sigma_{2}\right)_{I J} Y^{i} C Q_{J} \sim\left(1 ; \square, 3 ; 2 N_{f}-4,3 \frac{N_{f}-4}{N_{f}+4}\right) \\
& P^{[i j k]}=\frac{1}{3 !} Q_{I}^{T}\left(\sigma_{2}\right)_{I J} Y^{[i} Y^{j} Y^{k]} C Q_{J} \sim\left(1 ; \text { 日 }, 1 ; 2 N_{f}-12,5 \frac{N_{f}-4}{N_{f}+4}\right) \\
& R^{[i j k l]}=\frac{1}{4 !} Q_{I}^{T}\left(\sigma_{\mathrm{x}} \sigma_{2}\right)_{I J} \Gamma^{\mu} C Q_{J} Q_{K}^{T}\left(\sigma_{\mathrm{x}} \sigma_{2}\right)_{K L} \Gamma_{\mu} Y^{[i} Y^{j} V^{k} Y^{l]} C Q_{L} \sim\left(1 ; \text { 日 }, 1 ; 4 N_{f}-16,8 \frac{N_{f}-4}{N_{f}+4}\right) \\
& T_{\mathrm{X}}^{[i j k l m]}=\frac{1}{5 !} Q_{I}^{T}\left(\sigma_{\mathrm{x}} \sigma_{2}\right)_{I J} Y^{[i} Y^{j} Y^{k} Y^{l} Y^{m]} C Q_{J} \sim\left(1 ; \text { 目 }, 3 ; 2 N_{f}-20,7 \frac{N_{f}-4}{N_{f}+4}\right)
\end{aligned}
$$

where Greek, small Latin and large Latin letters respectively denote $S O(10)$ color, $S U\left(N_{f}\right)$ vector and $S U(2)$ spinor indices.

It is instructive to compare the number of these composite operators with the number of independent flat directions as a function of $N_{f}$. We perform this comparison in Table 2. Looking at the table's entries, we see that $K, M, N$ and $P$ account for all massless fields in the $S O(10)$ model with three or fewer vector flavors. On the other hand, the hadron count exceeds the needed number of composites by one when $N_{f}=4$. A single constraint must therefore exist among the hadron fields in this case. The precise quantum constraint relation is fixed by symmetry and the classical limit [35]. It appears in superpotential form as

$$
\begin{aligned}
W_{N_{f}=4}=X & {\left[4 R^{2}+12 K P_{i} M^{i j} P_{j}-36 N_{\mathrm{X}}^{i} N_{\mathrm{X}}^{j} P_{i} P_{j}-12 i \epsilon_{i_{1} i_{2} i_{3} i_{4}} \epsilon_{\mathrm{XYZ}} N_{\mathrm{X}}^{i_{1}} N_{\mathrm{Y}}^{i_{2}} N_{\mathrm{Z}}^{i_{3}} M^{i_{4} j} P_{j}\right.} \\
& +\epsilon_{i_{1} i_{2} i_{3} i_{4}} \epsilon_{j_{1} j_{2} j_{3} j_{4}}\left(2 K M^{i_{1} j_{1}} M^{i_{2} j_{2}} M^{i_{3} j_{3}} N_{\mathrm{X}}^{i_{4}} N_{\mathrm{X}}^{j_{4}}-9 N_{\mathrm{X}}^{i_{1}} N_{\mathrm{X}}^{j_{1}} N_{\mathrm{Y}}^{i_{2}} N_{\mathrm{Y}}^{j_{2}} M^{i_{3} j_{3}} M^{i_{4} j_{4}}\right) \\
& \left.-4 K^{2} \operatorname{det} M-\Lambda_{4}^{16}\right]
\end{aligned}
$$

4 We implicitly regard the 16 -dimensional $S O(10)$ spinor as the projection $Q=P_{-} Q_{32}$ where $Q_{32}$ denotes the 32 -dimensional spinor of $S O(11)$ and $P_{-}=\frac{1}{2}\left(1-\Gamma_{11}\right)$. The $32 \times 32$ Gamma matrices of $S O(10)$ along with its charge conjugation matrix $C$ come from the Clifford algebra of $S O(11)$. 


\begin{tabular}{|c|c|c|c|c|c|c|c|c|}
\hline$N_{f}$ & Hadrons & $K$ & $M$ & $N$ & $P$ & $R$ & $T$ & constraints \\
\hline 0 & 1 & 1 & & & & & & \\
\hline 1 & 5 & 1 & 1 & 3 & & & & \\
\hline 2 & 10 & 1 & 3 & 6 & & & & \\
\hline 3 & 17 & 1 & 6 & 9 & 1 & & & \\
\hline 4 & 27 & 1 & 10 & 12 & 4 & 1 & & -1 \\
\hline 5 & 37 & 1 & 15 & 15 & 10 & 5 & 3 & -12 \\
\hline
\end{tabular}

Table 2: Hadron degree of freedom count in the $S O(10)$ theory with two spinors

where the superfield $X$ represents a Lagrange multiplier. As a check on this result, one can Higgs the $S O(10)$ gauge group down to $S O(6) \simeq S U(4)$ by giving vevs $\left\langle V_{\mu}^{i}\right\rangle=v \delta_{\mu}^{i}$ to the four vectors. The two 16-dimensional spinor fields then split apart into four $4+\overline{4}$ pairs. After the $S O(10)$ hadrons are decomposed in terms of $S U(4)$ mesons and baryons, one finds that the quantum constraint in (2.7) properly reduces to the $N_{f}=N_{c}=4 \mathrm{SUSY}$ QCD relation $\operatorname{det} M_{4 \times 4}-B \bar{B}=\Lambda^{8}$ [35].

The hadron fields in the $N_{f}=5$ theory are restricted by 12 independent relations. These constraints are encoded within the equations of motion that follow from the $N_{f}=5$ superpotential

$$
\begin{aligned}
& W_{N_{f}=5}=\frac{1}{\Lambda_{5}^{15}}\left[12 K T_{\mathrm{X}} T_{\mathrm{X}}+36 i \epsilon_{\mathrm{XYZ}} N_{\mathrm{X}}^{i} N_{\mathrm{Y}}^{j} P_{i j} T_{\mathrm{Z}}-24 N_{\mathrm{X}}^{i} R_{i} T_{\mathrm{X}}+3 \epsilon^{i j k l m} P_{i j} P_{k l} R_{m}+4 R_{i} M^{i j} R_{j}\right. \\
& +6 K M^{i j} M^{k l} P_{i k} P_{j l}-36 M^{i j} N_{\mathrm{X}}^{k} N_{\mathrm{X}}^{l} P_{i k} P_{j l}-6 i \epsilon_{i_{1} i_{2} i_{3} i_{4} i_{5}} \epsilon_{\mathrm{XYZ}} N_{\mathrm{X}}^{i_{1}} N_{\mathrm{Y}}^{i_{2}} N_{\mathrm{z}}^{i_{3}} M^{i_{4} j} M^{i_{5} k} P_{j k} \\
& +\epsilon_{i_{1} i_{2} i_{3} i_{4} i_{5}} \epsilon_{j_{1} j_{2} j_{3} j_{4} j_{5}}\left(\frac{1}{2} K M^{i_{1} j_{1}} M^{i_{2} j_{2}} M^{i_{3} j_{3}} M^{i_{4} j_{4}} N_{\mathrm{X}}^{i_{5}} N_{\mathrm{X}}^{j_{5}}-3 N_{\mathrm{X}}^{i_{1}} N_{\mathrm{X}}^{j_{1}} N_{\mathrm{Y}}^{i_{2}} N_{\mathrm{Y}}^{j_{2}} M^{i_{3} j_{3}} M^{i_{4} j_{4}} M^{i_{5} j_{5}}\right) \\
& \left.-4 K^{2} \operatorname{det} M\right] .
\end{aligned}
$$

After varying this complicated expression, we observe that the origin $K=M=N=P=$ $R=T=0$ satisfies all equations of motion and lies on the quantum moduli space. Since the full global symmetry group remains unbroken at this point, all 't Hooft anomalies calculated within the microscopic and effective theories should agree. We find that the parton 
and hadron level $S U\left(N_{f}\right)^{3}, S U\left(N_{f}\right)^{2} U(1)_{Y}, S U\left(N_{f}\right)^{2} U(1)_{R}, S U(2)^{2} U(1)_{Y}, S U(2)^{2} U(1)_{R}$, $U(1)_{Y}, U(1)_{Y}^{3}, U(1)_{R}, U(1)_{R}^{3}, U(1)_{Y}^{2} U(1)_{R}$ and $U(1)_{R}^{2} U(1)_{Y}$ anomalies do indeed match when $N_{f}=5$ [36]. This nontrivial anomaly agreement strongly suggests that the effective theory contains only the composite fields in (2.6) and no additional colored or colorless massless degrees of freedom.

The $N_{Q}=2, N_{f}=5 S O(10)$ model is clearly analogous to $N_{f}=N_{c}+1$ SUSY QCD 35] which is commonly, though imprecisely, referred to as confining. The $S O(10)$ theory with $N_{Q}=2, N_{f}=6$ is similarly reminiscent of SUSY QCD with $N_{f}=N_{c}+2$ flavors, inasmuch as microscopic and macroscopic global anomalies fail to match and any effective superpotential would have to involve a branch cut. The anomaly mismatch cannot be remedied by introducing additional color-singlet fields without disrupting the $N_{f}=5$ results. Moreover, the $N_{f}=6$ model flows along a spinor flat direction to $G_{2}$ theory with six fundamentals which is known to exist within a nonabelian Coulomb phase [2]. We conclude that the $N_{f}=6 S O(10)$ theory cannot be represented in terms of gauge singlet operators in the far infrared. Instead, we expect that it resides within a new phase which possesses a dual description. As we shall see in the next section, this expectation is correct.

\section{The $S U\left(N_{f}-3\right) \times S p(2)$ dual}

Although no systematic field theory method currently exists for constructing nontrivial duals, some patterns have been found in special cases. For instance, partners to several models which possess tree level superpotentials [24,25, 37 40] have been uncovered following Kutasov's first example of SUSY QCD with adjoint matter [26]. Another class of dual pairs which exhibits certain trends stems from Pouliot's $G_{2}$ model with $N_{f}$ fields in the fundamental irrep [2]. The dual to this theory is based upon an $S U\left(N_{f}-3\right)$ gauge group, and its matter content includes a symmetric tensor. Counterparts to $S O(7), S O(8), S O(9)$ and $S O(10)$ generalizations of Pouliot's $G_{2}$ model are qualitatively similar [2,3, 27, 30]. Unlike the Kutasov-type dual pairs, the Pouliot-type examples can be arranged to have zero tree level superpotential on one side. Of course after having found such transformations, one can always choose to turn on some classical superpotential and study resulting dual pair deformations.

Our $S O(10)$ theory with two spinors (which we will refer to as the "electric" theory) reduces to Pouliot's $G_{2}$ model along a flat direction where both spinors acquire vevs. We consequently begin our search for a dual to the $N_{Q}=2$ model (which we will refer to as 
the "magnetic" theory) by looking for extensions of the $S U\left(N_{f}-3\right)$ counterpart to the $G_{2}$ theory. Since Higgsing the electric theory often induces mass decoupling on the magnetic side but leaves the color group unaltered, we hypothesize that $\widetilde{G}$ contains a local $S U\left(N_{f}-3\right)$ factor. We also presume that the $S O(10)$ model's full $S U\left(N_{f}\right) \times S U(2) \times U(1)_{Y} \times U(1)_{R}$ global symmetry is realized at short as well as long distance scales in the dual. Our initial guess for the magnetic symmetry group is thus

$$
\widetilde{G}=\left[S U\left(N_{f}-3\right)\right]_{\text {local }} \times\left[S U\left(N_{f}\right) \times S U(2) \times U(1)_{Y} \times U(1)_{R}\right]_{\text {global }}
$$

Following the examples of all previously constructed Pouliot-type duals, we introduce quark matter fields which transform under $\widetilde{G}$ as

$$
q_{i}^{\alpha} \sim\left(\square ; \bar{\square}, 1 ; 2 \frac{N_{f}-6}{N_{f}-3}, \frac{5\left(N_{f}-4\right)}{\left(N_{f}-3\right)\left(N_{f}+4\right)}\right) .
$$

The hypercharge and R-charge assignments have been chosen so that the magnetic baryon $p=q^{N_{f}-3}$ naturally maps onto the electric baryon $P$ in (2.6). If we also incorporate the composite $M^{(i j)}$ and $N_{\mathrm{x}}^{i}$ electric mesons into the magnetic theory as elementary fields $m^{(i j)}$ and $n_{\mathrm{x}}^{i}$, we find that the $S U\left(N_{f}\right)^{3}, S U\left(N_{f}\right)^{2} U(1)_{Y}$ and $S U\left(N_{f}\right)^{2} U(1)_{R}$ global 't Hooft anomalies match between the $S O(10)$ model and its dual. This nontrivial anomaly agreement suggests that we have properly identified all magnetic matter fields which transform nontrivially under the $S U\left(N_{f}\right)$ global symmetry group.

We next introduce into the magnetic theory a symmetric tensor field

$$
s_{\alpha \beta} \sim\left(\bar{\square} ; 1,1 ; \frac{4 N_{f}}{N_{f}-3}, 2 \frac{3 N_{f}-4}{\left(N_{f}-3\right)\left(N_{f}+4\right)}\right),
$$

dual antiquarks

$$
\bar{q}_{\alpha}^{\mathrm{x}} \sim\left(\bar{\square} ; 1,3 ;-2 N_{f} \frac{N_{f}-4}{N_{f}-3},-\frac{N_{f}^{2}-18 N_{f}+40}{\left(N_{f}-3\right)\left(N_{f}+4\right)}\right)
$$

and superpotential interaction terms

$$
W_{\mathrm{mag}}=\frac{1}{\mu_{1}^{2}} m^{(i j)} q_{i}^{\alpha} s_{\alpha \beta} q_{j}^{\beta}+\frac{1}{\mu_{2}^{2}} n_{\mathrm{x}}^{i} q_{i}^{\alpha} \bar{q}_{\alpha}^{\mathrm{x}}
$$

All of these items are common ingredients in Pouliot-type duals. The abelian quantum numbers for $s$ and $\bar{q}$ are fixed by requiring invariance of both terms in $W_{\text {mag }}$ under $\widetilde{G}$. 
With these dual matter fields now in hand, we find that the electric baryons 0

$$
\begin{aligned}
B_{0}^{\left[i_{1} \cdots i_{10}\right]} & =\epsilon^{\mu_{1} \cdots \mu_{10}} V_{\mu_{1}}^{i_{1}} \cdots V_{\mu_{10}}^{i_{10}} \\
B_{1}^{a\left[i_{1} \cdots i_{8}\right]} & =\epsilon^{\mu_{1} \cdots \mu_{10}} V_{\mu_{1}}^{i_{1}} \cdots V_{\mu_{8}}^{i_{8}} W_{\mu_{9} \mu_{10}}^{a} \\
B_{2}^{\left[i_{1} \cdots i_{6}\right]} & =\epsilon^{\mu_{1} \cdots \mu_{10}} \epsilon_{a b} V_{\mu_{1}}^{i_{1}} \cdots V_{\mu_{6}}^{i_{6}} W_{\mu_{7} \mu_{8}}^{a} W_{\mu_{9} \mu_{10}}^{b}
\end{aligned}
$$

can be mapped onto the magnetic operators

$$
\begin{aligned}
b_{0}^{\left[i_{1} \cdots i_{10}\right]}= & \epsilon^{i_{1} \cdots i_{N_{f}}} \epsilon^{\alpha_{1} \cdots \alpha_{N_{f}-3}} \epsilon_{\mathrm{XYZ}} \epsilon_{a b} \\
& (s q)_{\alpha_{1} i_{11}} \cdots(s q)_{\alpha_{N_{f}-10} i_{N_{f}}}\left(s \widetilde{w}^{a}\right)_{\alpha_{N_{f}-9} \alpha_{N_{f}-8}}\left(s \widetilde{w}^{b}\right)_{\alpha_{N_{f}-7} \alpha_{N_{f}-6}} \bar{q}_{\alpha_{N_{f}-5}}^{\mathrm{X}} \bar{q}_{\alpha_{N_{f}-4}}^{\mathrm{Y}} \bar{q}_{\alpha_{N_{f}-3}}^{\mathrm{Z}} \\
b_{1}^{a\left[i_{1} \cdots i_{8}\right]}= & \epsilon^{i_{1} \cdots i_{N_{f}}} \epsilon^{\alpha_{1} \cdots \alpha_{N_{f}-3}} \epsilon_{\mathrm{XYZ}} \\
& (s q)_{\alpha_{1} i_{9}} \cdots(s q)_{\alpha_{N_{f}-8} i_{N_{f}}}\left(s \widetilde{w}^{a}\right)_{\alpha_{N_{f}-7} \alpha_{N_{f}-6}} \bar{q}_{\alpha_{N_{f}-5}}^{\mathrm{X}} \bar{q}_{\alpha_{N_{f}-4}}^{\mathrm{Y}} \bar{q}_{\alpha_{N_{f}-3}}^{\mathrm{Z}} \\
b_{2}^{a\left[i_{1} \cdots i_{6}\right]}= & \epsilon^{i_{1} \cdots i_{N_{f}}} \epsilon^{\alpha_{1} \cdots \alpha_{N_{f}-3}} \epsilon_{\mathrm{XYZ}}(s q)_{\alpha_{1} i_{7}} \cdots(s q)_{\alpha_{N_{f}-6} i_{N_{f}}} \bar{q}_{\alpha_{N_{f}-5}}^{\mathrm{X}} \bar{q}_{\alpha_{N_{f}-4}}^{\mathrm{Y}} \bar{q}_{\alpha_{N_{f}-3}}^{\mathrm{Z}} .
\end{aligned}
$$

The more exotic combinations of $S O(10)$ vectors, spinors and gluons

$$
\begin{aligned}
C_{0 \mathrm{X}}^{\left[i_{1} \cdots i_{9}\right]} & =\epsilon^{\mu_{1} \cdots \mu_{10}} V_{\mu_{1}}^{i_{1}} \cdots V_{\mu_{9}}^{i_{9}} Q_{I}^{T}\left(\sigma_{\mathrm{X}} \sigma_{2}\right)_{I J} \Gamma_{\mu_{10}} C Q_{J} \\
C_{1 \mathrm{X}}^{a\left[i_{1} \cdots i_{7}\right]} & =\epsilon^{\mu_{1} \cdots \mu_{10}} V_{\mu_{1}}^{i_{1}} \cdots V_{\mu_{7}}^{i_{7}} W_{\mu_{8} \mu_{9}}^{a} Q_{I}^{T}\left(\sigma_{\mathrm{X}} \sigma_{2}\right)_{I J} \Gamma_{\mu_{10}} C Q_{J} \\
C_{2}{ }_{2}^{\left[i_{1} \cdots i_{5}\right]} & =\epsilon^{\mu_{1} \cdots \mu_{10}} \epsilon_{a b} V_{\mu_{1}}^{i_{1}} \cdots V_{\mu_{5}}^{i_{5}} W_{\mu_{6} \mu_{7}}^{a} W_{\mu_{8} \mu_{9}}^{b} Q_{I}^{T}\left(\sigma_{\mathrm{X}} \sigma_{2}\right)_{I J} \Gamma_{\mu_{10}} C Q_{J}
\end{aligned}
$$

may similarly be identified with the dual composites

$$
\begin{aligned}
c_{0 \mathrm{X}}^{\left[i_{1} \cdots i_{9}\right]} & =\epsilon^{i_{1} \cdots i_{N_{f}}} \epsilon^{\alpha_{1} \cdots \alpha_{N_{f}-3}} \epsilon_{\mathrm{XYZ}} \epsilon_{a b} \\
& (s q)_{\alpha_{1} i_{10}} \cdots(s q)_{\alpha_{N_{f}-9} i_{N_{f}}}\left(s \widetilde{w}^{a}\right)_{\alpha_{N_{f}-8} \alpha_{N_{f}-7}}\left(s \widetilde{w}^{b}\right)_{\alpha_{N_{f}-6} \alpha_{N_{f}-5}} \bar{q}_{\alpha_{N_{f}-4}}^{\mathrm{Y}} \bar{q}_{\alpha_{N_{f}-3}}^{\mathrm{Z}} \\
c_{1 \mathrm{x}}^{a\left[i_{1} \cdots i_{7}\right]} & =\epsilon^{i_{1} \cdots i_{N_{f}}} \epsilon^{\alpha_{1} \cdots \alpha_{N_{f}-3}} \epsilon_{\mathrm{XYZ}}(s q)_{\alpha_{1} i_{8}} \cdots(s q)_{\alpha_{N_{f}-7} i_{N_{f}}}\left(s \widetilde{w}^{a}\right)_{\alpha_{N_{f}-6} \alpha_{N_{f}-5}} \bar{q}_{\alpha_{N_{f}-4}}^{\mathrm{Y}} \bar{q}_{\alpha_{N_{f}-3}}^{\mathrm{Z}} \\
c_{2 \mathrm{x}}^{\left[i_{1} \cdots i_{5}\right]} & =\epsilon^{i_{1} \cdots i_{N_{f}}} \epsilon^{\alpha_{1} \cdots \alpha_{N_{f}-3}} \epsilon_{\mathrm{XYZ}}(s q)_{\alpha_{1} i_{6}} \cdots(s q)_{\alpha_{N_{f}-5} i_{N_{f}}} \bar{q}_{\alpha_{N_{f}-4}}^{\mathrm{Y}} \bar{q}_{\alpha_{N_{f}-3}}^{\mathrm{Z}} .
\end{aligned}
$$

The overall consistency of these gauge invariant operator identifications is encouraging.

Our construction of the dual to the $S O(10)$ theory with two spinors has so far closely mimicked that for the dual to $S O(10)$ with one spinor [3]. But as other investigators have recently observed [29], continuing in this direction ultimately leads to a dead-end. We

5 The two anticommuting fermionic field strength tensors in $B_{2}, C_{2}, b_{0}$ and $c_{0}$ must be contracted together into antisymmetric spin- 0 combinations in order for these electric and magnetic baryons not to vanish. 
cannot find a magnetic theory based upon the symmetry group in (3.1) for which all local anomalies cancel, all global anomalies match and all composite operators map. We must therefore relax some assumption in order to make further progress. After exploring several possibilities, we are forced to conclude that the magnetic gauge group is not simple. We consequently expand our search by looking for a dual with a product color group.

The simplest generalization which retains the previous desirable features while overcoming the above-mentioned difficulties has symmetry group 6

$$
\widetilde{G}=\left[S U\left(N_{f}-3\right) \times S p(2)\right]_{\text {local }} \times\left[S U\left(N_{f}\right) \times S U(2) \times U(1)_{Y} \times U(1)_{R}\right]_{\text {global }}
$$

superfield matter content

$$
\begin{aligned}
q_{i}^{\alpha} & \sim\left(\square, 1 ; \bar{\square}, 1 ; 2 \frac{N_{f}-6}{N_{f}-3}, \frac{5\left(N_{f}-4\right)}{\left(N_{f}-3\right)\left(N_{f}+4\right)}\right) \\
{q^{\prime}}_{I}^{\alpha \dot{\alpha}} & \sim\left(\square, 2 ; 1,2 ;-\frac{2 N_{f}}{N_{f}-3}, \frac{\left(N_{f}+2\right)\left(N_{f}-4\right)}{\left(N_{f}-3\right)\left(N_{f}+4\right)}\right) \\
\bar{q}_{\alpha}^{\mathrm{X}} & \sim\left(\bar{\square}, 1 ; 1,3 ;-2 N_{f} \frac{N_{f}-4}{N_{f}-3},-\frac{N_{f}^{2}-18 N_{f}+40}{\left(N_{f}-3\right)\left(N_{f}+4\right)}\right) \\
s_{\alpha \beta} & \sim\left(\bar{\square}, 1 ; 1,1 ; \frac{4 N_{f}}{N_{f}-3}, 2 \frac{3 N_{f}-4}{\left(N_{f}-3\right)\left(N_{f}+4\right)}\right) \\
t_{I}^{\dot{\alpha}} & \sim\left(1,2 ; 1,2 ; 2 N_{f}, 2 \frac{N_{f}-4}{N_{f}+4}\right) \\
m^{(i j)} & \sim\left(1,1 ; \square, 1 ;-8,2 \frac{N_{f}-4}{N_{f}+4}\right) \\
n_{\mathrm{x}}^{i} & \sim\left(1,1 ; \square, 3 ; 2 N_{f}-4,3 \frac{N_{f}-4}{N_{f}+4}\right)
\end{aligned}
$$

and tree level superpotential

$$
W_{\mathrm{mag}}=\frac{1}{\mu_{1}^{2}} m^{(i j)} q_{i}^{\alpha} s_{\alpha \beta} q_{j}^{\beta}+\frac{1}{\mu_{2}^{2}} n_{\mathrm{x}}^{i} q_{i}^{\alpha} \bar{q}_{\alpha}^{\mathrm{x}}+\lambda_{1} \epsilon_{\dot{\alpha} \dot{\beta}} \epsilon_{I}^{I J}{q_{I}^{\prime}}_{I}^{\alpha \dot{\alpha}} s_{\alpha \beta}{q_{J}^{\prime \beta}}_{J}^{\dot{\beta}}+\lambda_{2} \epsilon_{\dot{\alpha} \dot{\beta}} q_{I}^{\prime \alpha \dot{\alpha}}\left(\sigma_{\mathrm{x}} \sigma_{2}\right)^{I J} \bar{q}_{\alpha}^{\mathrm{x}} t_{J}^{\dot{\beta}} .
$$

Several points about this product dual should be noted. Firstly, the $S U\left(N_{f}-3\right)^{3}$, $S U\left(N_{f}-3\right)^{2} U(1)_{Y}, S U\left(N_{f}-3\right)^{2} U(1)_{R}, S p(2)^{2} U(1)_{Y}$, and $S p(2)^{2} U(1)_{R}$ anomalies vanish, and an even number of doublets transform under the color $S p(2)$ [41]. The magnetic gauge group and global abelian hypercharge and R-charge symmetries are consequently

6 We remind the reader that in our conventions $S p(2) \simeq S U(2)$. We use dotted Greek letters to denote $S p(2)$ color indices. 
free of all perturbative and nonperturbative anomalies like their electric counterparts. All anomalies involving only global symmetries also match between the electric and magnetic theories for $N_{f} \geq 5$. Secondly, we treat the colored partons in this theory as canonically normalized, but we set the engineering mass dimensions of the colorless $m^{(i j)}$ and $n_{\mathrm{x}}^{i}$ fields equal to those of their electric counterparts. In order for the magnetic superpotential to have dimension three, the first two nonrenormalizable terms in (3.12) must be multiplied by dimensionful prefactors $\mu_{1}^{-2}$ and $\mu_{2}^{-2}$. On the other hand, the $\lambda_{1}$ and $\lambda_{2}$ coefficients in the third and fourth terms of $W_{\text {mag }}$ are dimensionless. For simplicity, we set all these prefactors equal to unity from here on. Finally, it is instructive to count the number of constraints on abelian charge assignments which determines how many nonanomalous $U(1)$ factors appear within the magnetic global symmetry group. We start with the 7 fermionic components of the $q, q^{\prime}, \bar{q}, s, t, m$, and $n$ matter superfields along with the $S U\left(N_{f}-3\right)$ gluino $\tilde{g}$ and $S p(2)$ wino $\tilde{w}$. Requiring the $S U\left(N_{f}-3\right)^{2} U(1)$ and $S p(2)^{2} U(1)$ anomalies to vanish imposes two conditions on these fermions' charges. We next recall that fermion-sfermion-gaugino interactions in the Kahler potential tie together the global quantum numbers for $\tilde{g}$ and $\tilde{w}$. The terms in the magnetic superpotential (3.12) impose 4 more conditions on abelian charge assignments. We thus find that the magnetic dual possesses $9-2-1-4=2$ independent global $U(1)$ symmetries which agrees with the electric theory.

The dual pair's phase structure represents an important dynamical issue. The Wilsonian beta function coefficients for the two gauge groups in $\widetilde{G}$ are given by $\tilde{b}_{0} S U\left(N_{f}-3\right)=$ $2 N_{f}-12$ and ${\tilde{b_{0}}}^{S p(2)}=8-N_{f}$. The $S U\left(N_{f}-3\right)$ factor is asymptotically free for $N_{f} \geq 7$, while the $S p(2)$ factor is asymptotically free for $N_{f} \leq 7$. Since there is no value of $N_{f}$ for which both are free in the infrared, the $S O(10)$ theory does not possess a free magnetic phase. Instead, it exists at the origin of moduli space in a nonabelian Coulomb phase for $6 \leq N_{f} \leq 19$ vector flavors. For $N_{f} \geq 20$, the magnetic theory flows to the weakly coupled $S O(10)$ theory at long distance scales. The absence of a free magnetic phase is a common feature in all similar dual pairs [2, 3, 27 30].

We next construct maps between gauge invariant operators in the $S O(10)$ and $S U\left(N_{f}-3\right) \times S p(2)$ theories. We have already matched several operators in (3.6)-(3.9), but there are many others to consider. We first identify the composites

$$
\begin{aligned}
k & =\epsilon_{\dot{\alpha} \dot{\beta}} \epsilon^{I J} t_{I}^{\dot{\alpha}} t_{J}^{\dot{\beta}} \\
p^{\left[i_{1} i_{2} i_{3}\right]} & =\epsilon^{i_{1} \cdots i_{N_{f}}} \epsilon_{\alpha_{1} \cdots \alpha_{N_{f}-3}} q_{i_{4}}^{\alpha_{1}} \cdots q_{i_{N_{f}}^{\alpha_{N_{f}-3}}}
\end{aligned}
$$




$$
\begin{aligned}
r^{\left[i_{1} i_{2} i_{3} i_{4}\right]} & =\epsilon^{i_{1} \cdots i_{N_{f}}} \epsilon_{\alpha_{1} \cdots \alpha_{N_{f}-3}} \epsilon_{\dot{\alpha} \dot{\beta}} \epsilon_{I J} q_{i_{5}}^{\alpha_{1}} \cdots q_{i_{N_{f}}}^{\alpha_{N_{f}-4}}{ }^{\prime^{\prime}}{ }_{I}^{\alpha_{N_{f}-3} \dot{\alpha}} t_{J}^{\dot{\beta}} \\
t_{\mathrm{X}}^{\left[i_{1} i_{2} i_{3} i_{4} i_{5}\right]} & =\epsilon^{i_{1} \cdots i_{N_{f}}} \epsilon_{\alpha_{1} \cdots \alpha_{N_{f}-3}} \epsilon_{\dot{\alpha} \dot{\beta}}\left(\sigma_{\mathrm{X}} \sigma_{2}\right)_{I J} q_{i_{6}}^{\alpha_{1}} \cdots q_{i_{N_{f}}}^{\alpha_{N_{f}-5}} q^{\prime{ }^{\prime}{ }_{N_{f}-4} \dot{\alpha}}{ }^{\alpha^{\prime}{ }_{J}^{\alpha_{N_{f}-3} \dot{\beta}}}
\end{aligned}
$$

as partners to $K, P, R$ and $T$ in (2.6). These magnetic hadrons share exactly the same quantum numbers as their electric theory counterparts. In particular, their transformation rules under the global $S U(2)$ which rotates the two spinors on the electric side are fixed once we form gauge invariant combinations of the dual matter fields. Other chiral operators besides those which act as moduli space coordinates in the confining phase can also be mapped. For example, the exotic $S O(10)$ invariants

$$
\begin{aligned}
& U=\epsilon_{a b} Q_{1} \Gamma^{\left[\mu_{1} \mu_{2} \mu_{3}\right]} Q_{2} Q_{1} \Gamma^{\left[\mu_{1} \nu_{2} \nu_{3}\right]} Q_{2} W_{\mu_{2} \nu_{2}}^{a} W_{\mu_{3} \nu_{3}}^{b} \\
& V=\epsilon_{a b} Q_{1} \Gamma^{\left[\mu_{1} \mu_{2} \mu_{3}\right]} Q_{2} Q_{1} \Gamma^{\left[\mu_{1} \mu_{2} \nu_{3}\right]} Q_{2} W_{\mu_{3} \sigma}^{a} W_{\nu_{3} \sigma}^{b}
\end{aligned}
$$

are identified with linear combinations of the $S U\left(N_{f}-3\right) \times S p(2)$ singlets

$$
\begin{aligned}
& u=\operatorname{det} s \\
& v=\epsilon_{\dot{\alpha} \dot{\beta}} \epsilon_{\dot{\gamma} \dot{\delta}} \epsilon^{I J} \epsilon^{K L} q_{I}^{\prime \alpha \dot{\alpha}} t_{J}^{\dot{\beta}} s_{\alpha \beta} q_{K}^{\prime \beta \dot{\gamma}^{\prime}} t_{L}^{\dot{\delta}} .
\end{aligned}
$$

We should point out that det $s$ does not appear anywhere within the magnetic superpotential (3.12). This feature of our product dual represents an interesting departure from previously studied Pouliot-type models. Since $u=\operatorname{det} s$ is not rendered redundant by equations of motion, it must match onto some linear combination of the primary $U$ and $V$ electric composites. Further details on mapping these $Q^{4} W^{2}$ operators are presented in Appendix A.

Given that det $s$ does not appear in the $S U\left(N_{f}-3\right) \times S p(2)$ theory's superpotential but does enter into $W_{\text {mag }}$ in Pouliot's $S U\left(N_{f}-3\right)$ dual to the $G_{2}$ model, we should inquire how this term arises when we deform the former into the latter. Recall from (2.4) that generic expectation values for the two 16-dimensional spinors $Q_{1}$ and $Q_{2}$ break the $S O(10)$ color group down to $G_{2}$. This symmetry breaking can alternatively be viewed as resulting from a large expectation value for the composite operator $K \sim\left(Q_{1} Q_{2}\right)^{2}$ :

$$
\langle K\rangle=a^{4} \gg \Lambda_{S O(10)}^{4} .
$$

The mapping in (3.13a) then implies that the dual parton field $t$ develops a nonzero vev which can be rotated into the form $\left\langle t_{I}^{\dot{\alpha}}\right\rangle=a^{2} \epsilon_{I}^{\dot{\alpha}}$. This condensate for $t$ gives mass to the 
$q^{\prime}$ and $\bar{q}$ matter fields via the last term in (3.12). Once heavy degrees of freedom are integrated out, the tree level magnetic superpotential reduces to

$$
W_{\mathrm{mag}} \rightarrow m^{\prime(i j)} q_{i}^{\alpha} s_{\alpha \beta} q_{j}^{\beta}-2 q_{0}^{\alpha} s_{\alpha \beta} q_{0}^{\beta}
$$

where $m^{\prime(i j)} \equiv m^{(i j)}-\frac{1}{2 a^{4}} n_{\mathrm{x}}^{i} n_{\mathrm{x}}^{j}$ and $q_{0}^{\alpha}$ denotes the only component of ${q_{I}^{\prime \alpha \dot{\alpha}}}_{\text {which does }}$ not grow massive. The vev for $t$ also completely breaks the magnetic $S p(2)$ color group. As a result, instanton effects generate the dynamical superpotential 420

$$
W_{\mathrm{dyn}}=\frac{\Lambda_{S p(2)}^{8-N_{f}}}{\langle K\rangle} \operatorname{det} s .
$$

After combining this quantum contribution with the classical terms in (3.17), we reproduce the total superpotential in Pouliot's dual to the $G_{2}$ model along with its gauge group and matter content [2]. This successful recovery of an old dual from our new one constitutes an important consistency check.

It is interesting to explore other deformations of the $N_{Q}=2 S O(10)$ model which yield novel dual pairs. We can find magnetic descriptions for a large number of electric theories by flowing along various vector and spinor flat directions as illustrated in fig. 11. For example, we sketch the derivation of a dual to an $S O(7)$ model with $N_{f}$ spinors and one vector. We first Higgs the $S O(10)$ gauge group down to $S O(7)$ by turning on a vev for $N_{+}^{N_{f}} \equiv N_{1}^{N_{f}}+i N_{2}^{N_{f}}$. We next give mass to all singlets not eaten by the superHiggs mechanism. The resulting deformed electric theory becomes

$$
G=S O(7)_{\text {local }} \times\left[S U\left(N_{f}\right) \times U(1)_{Y} \times U(1)_{R}\right]_{\text {global }}
$$

with superfield matter content

$$
\begin{gathered}
V_{\mu} \sim\left(7 ; 1 ; N_{f}, \frac{N_{f}-4}{N_{f}+1}\right) \\
Q_{I}^{A} \sim\left(8 ; \square ;-1, \frac{N_{f}-4}{N_{f}+1}\right) .
\end{gathered}
$$

7 The nonperturbative mechanism underlying (3.18) is the same as that which produces the determinant within the $N_{f}=N_{c}+1$ SUSY QCD superpotential $W=(B M \bar{B}-\operatorname{det} M) / \Lambda^{2 N_{c}-1}$ [35]. When the magnetic $S U(2)$ gauge group in the $N_{f}=N_{c}+2$ theory is Higgsed, the $\operatorname{det} M$ term is generated by a weak coupling instanton process [1]. 
On the magnetic side of the dual pair, we rename all matter fields that previously transformed as doublets or triplets under the global $S U(2)$ in (3.10) which no longer exists in the new $S O(7)$ theory. Then after inserting $\left\langle n_{+}^{N_{f}}\right\rangle$ into $W_{\text {mag }}$ and eliminating heavy degrees of freedom by solving their equations of motion, we find that the deformed dual has symmetry group

$$
\widetilde{G}=\left[S U\left(N_{f}-3\right) \times S p(2)\right]_{\text {local }} \times\left[S U\left(N_{f}-1\right) \times U(1)_{N_{f}} \times U(1)_{Y} \times U(1)_{R}\right]_{\text {global }}
$$

matter content

$$
\begin{aligned}
& q_{i}^{\alpha} \sim\left(\square, 1 ; \bar{\square}, \frac{2}{N_{f}-3}, \frac{N_{f}-2}{N_{f}-3}, \frac{3\left(N_{f}-4\right)}{\left(N_{f}+1\right)\left(N_{f}-3\right)}\right) \\
& q_{1}^{\alpha \dot{\alpha}} \sim\left(\square, 2 ; 1 ; \frac{N_{f}-1}{N_{f}-3},-\frac{1}{2} \frac{N_{f}^{2}-3 N_{f}-2}{N_{f}-3}, \frac{1}{2} \frac{\left(N_{f}+3\right)\left(N_{f}-4\right)}{\left(N_{f}+1\right)\left(N_{f}-3\right)}\right) \\
& q_{2}^{\prime \alpha \dot{\alpha}} \sim\left(\square, 2 ; 1 ; \frac{N_{f}-1}{N_{f}-3}, \frac{1}{2} \frac{\left(N_{f}-2\right)\left(N_{f}-1\right)}{N_{f}-3}, \frac{3}{2} \frac{\left(N_{f}-1\right)\left(N_{f}-4\right)}{\left(N_{f}+1\right)\left(N_{f}-3\right)}\right) \\
& \bar{q}_{\alpha}^{+} \sim\left(\bar{\square}, 1 ; 1 ; \frac{\left(N_{f}-4\right)\left(N_{f}-1\right)}{N_{f}-3},-\frac{\left(N_{f}-2\right)^{2}}{N_{f}-3},-\frac{N_{f}^{2}-14 N_{f}+30}{\left(N_{f}+1\right)\left(N_{f}-3\right)}\right) \\
& \bar{q}_{\alpha}^{3} \sim\left(\bar{\square}, 1 ; 1 ; \frac{\left(N_{f}-4\right)\left(N_{f}-1\right)}{N_{f}-3}, \frac{N_{f}-4}{N_{f}-3}, \frac{7 N_{f}-18}{\left(N_{f}+1\right)\left(N_{f}-3\right)}\right) \\
& s_{\alpha \beta} \sim\left(\bar{\square}, 1 ; 1 ;-2 \frac{N_{f}-1}{N_{f}-3},-\frac{2}{N_{f}-3}, \frac{4 N_{f}-6}{\left(N_{f}+1\right)\left(N_{f}-3\right)}\right) \\
& t_{1}^{\dot{\alpha}} \sim\left(1,2 ; 1 ; 1-N_{f},-\frac{N_{f}+2}{2}, \frac{1}{2} \frac{N_{f}-4}{N_{f}+1}\right) \\
& t_{2}^{\dot{\alpha}} \sim\left(1,2 ; 1 ; 1-N_{f}, \frac{N_{f}-2}{2}, \frac{3}{2} \frac{N_{f}-4}{N_{f}+1}\right) \\
& m^{(i j)} \sim\left(1,1 ; \square ; 2,-2,2 \frac{N_{f}-4}{N_{f}+1}\right) \\
& n_{3}^{i} \sim\left(1,1 ; \square ; 2-N_{f},-2,2 \frac{N_{f}-4}{N_{f}+1}\right) \\
& n_{-}^{i} \sim\left(1,1 ; \square ; 2-N_{f}, N_{f}-2,3 \frac{N_{f}-4}{N_{f}+1}\right) \\
& n_{-}^{N_{f}} \sim\left(1,1 ; 1 ; 0,2 N_{f}, 2 \frac{N_{f}-4}{N_{f}+1}\right)
\end{aligned}
$$

and tree level superpotential

$$
\begin{gathered}
W_{\text {mag }}=m^{(i j)} q_{i}^{\alpha} s_{\alpha \beta} q_{j}^{\beta}+n_{-}^{i} q_{i}^{\alpha} \bar{q}_{\alpha}^{+}+n_{3}^{i} q_{i}^{\alpha} \bar{q}_{\alpha}^{3}+\epsilon_{\dot{\alpha} \dot{\beta}} n_{-}^{N_{f}} \bar{q}_{\alpha}^{+} q_{1}^{\prime \alpha \dot{\alpha}} t_{1}^{\dot{\beta}}+\epsilon_{\dot{\alpha} \dot{\beta}} q_{1}^{\alpha \dot{\alpha}} s_{\alpha \beta} q_{2}^{\prime \beta \dot{\beta}} \\
+\epsilon_{\dot{\alpha} \dot{\beta}} \bar{q}_{\alpha}^{3}\left(q_{1}^{\prime \alpha \dot{\alpha}} t_{2}^{\dot{\beta}}+q_{2}^{\prime \alpha \dot{\alpha}} t_{1}^{\dot{\beta}}\right)+\epsilon_{\dot{\alpha} \dot{\beta}} \bar{q}_{\alpha}^{+} q_{2}^{\prime \alpha \dot{\alpha}} t_{2}^{\dot{\beta}} .
\end{gathered}
$$


Only an $S U\left(N_{f}-1\right) \times U(1)_{N_{f}}$ subgroup of the global $S U\left(N_{f}\right)$ that rotates the spinors in the $S O(7)$ theory is realized at short distance scales in this dual. The subgroup's origin can be traced from the flows in fig. 1. One of the two 16-dimensional spinor fields is eaten when $S O(10)$ is Higgsed down to $S O(7)$, while the second breaks apart as $16 \rightarrow 8+7+1$. Nonrenormalizable terms pick out the lone 8 from the 16 and prevent its mixing with the $N_{f}-1$ other spinors that come from the 10's. The full $S U\left(N_{f}\right)$ symmetry is restored in the electric theory only in the far infrared where all nonrenormalizable interactions are negligible. On the magnetic side, the $S U\left(N_{f}\right)$ symmetry is realized in the infrared only at the quantum level. Similar accidental restoration of global symmetries was first observed in $S U(2)$ duality [1] and has been discussed in the recent literature [29,43].

As a final check on the $S U\left(N_{f}-3\right) \times S p(2)$ counterpart to the $N_{Q}=2 \mathrm{SO}(10)$ model, we investigate its entry into the confining phase when $N_{f}=5$. In this case, the dual gauge group reduces to $\widetilde{G}_{\text {local }}=S U(2)_{L} \times S p(2)_{R}$ where we have appended left and right labels onto the two group factors in order to distinguish them. The $S U(2)_{L}$ and $S p(2)_{R}$ gauge groups are respectively infrared and asymptotically free at high energies. The latter grows strong at a scale $\Lambda_{R}$ and confines the 6 doublets $q^{\prime}$ and $t$ into the antisymmetric matrix 35]

$$
M_{R}=\left(\begin{array}{cc}
{q_{I}^{\prime}}_{I}^{\alpha \dot{\alpha}}\left(\sigma_{2}\right)_{\dot{\alpha} \dot{\beta}} q_{J}^{\prime \beta \dot{\beta}} & q_{I}^{\prime \alpha \dot{\alpha}}\left(\sigma_{2}\right)_{\dot{\alpha} \dot{\beta}} t_{J}^{\dot{\beta}} \\
t_{J}^{\dot{\beta}}\left(\sigma_{2}^{T}\right)_{\dot{\beta} \dot{\alpha}} q_{I}^{\alpha \dot{\alpha}} & t_{I}^{\dot{\alpha}}\left(\sigma_{2}\right)_{\dot{\alpha} \dot{\beta}} t_{J}^{\dot{\beta}}
\end{array}\right)
$$

The mesons

$$
\begin{aligned}
k & =-\left(\sigma_{2}\right)_{I J} t_{I}^{\dot{\alpha}}\left(\sigma_{2}\right)_{\dot{\alpha} \dot{\beta}} t_{J}^{\dot{\beta}} \\
\left(s_{R}\right)^{(\alpha \beta)} & =\left(\sigma_{2}\right)_{I J} q_{I}^{\prime \alpha \dot{\alpha}}\left(\sigma_{2}\right)_{\dot{\alpha} \dot{\beta}} q_{J}^{\prime \beta \dot{\beta}} \\
\left(t_{R}\right)_{\mathrm{x}}^{[\alpha \beta]} & =i\left(\sigma_{\mathrm{x}} \sigma_{2}\right)_{I J}{q_{I}^{\prime \alpha}}^{\alpha \dot{\alpha}}\left(\sigma_{2}\right)_{\dot{\alpha} \dot{\beta}} q_{J}^{\prime \beta \dot{\beta}} \\
u_{R}^{\alpha} & =\left(\sigma_{2}\right)_{I J} q_{I}^{\prime \alpha \dot{\alpha}}\left(\sigma_{2}\right)_{\dot{\alpha} \dot{\beta}} t_{J}^{\dot{\beta}} \\
\left(v_{R}\right)_{\mathrm{x}}^{\alpha} & =i\left(\sigma_{\mathrm{x}} \sigma_{2}\right)_{I J}{q_{I}^{\prime}}^{\prime \alpha \dot{\alpha}}\left(\sigma_{2}\right)_{\dot{\alpha} \dot{\beta}} t_{J}^{\dot{\beta}}
\end{aligned}
$$

along with the $S p(2)_{R}$ invariants in (3.22) then represent the active matter degrees of freedom. Their dynamics are governed by the superpotential $W_{R}=W_{\text {tree }}+W_{\mathrm{dyn}}^{R}$ where

$$
W_{\text {tree }}=m^{(i j)} q_{i}^{\alpha} s_{\alpha \beta} q_{j}^{\beta}+n_{\mathrm{x}}^{i} q_{i}^{\alpha} \bar{q}_{\alpha}^{\mathrm{x}}-s_{\alpha \beta}\left(s_{R}\right)^{\alpha \beta}+\bar{q}_{\alpha}^{\mathrm{x}}\left(v_{R}\right)_{\mathrm{x}}^{\alpha}
$$

and

$$
\begin{aligned}
W_{\mathrm{dyn}}^{R}=\frac{\operatorname{Pf} M_{R}}{\Lambda_{R}{ }^{3}}= & \frac{1}{8 \Lambda_{R}{ }^{3}}\left\{k\left(\operatorname{Pf} t_{R}^{\mathrm{x}}\right)\left(\operatorname{Pf} t_{R}^{\mathrm{x}}\right)-k \operatorname{det} s_{R}-u_{R}^{T} \sigma_{2} s_{R} \sigma_{2} u_{R}-\left(v_{R}\right)_{\mathrm{X}}^{T} \sigma_{2} s_{R} \sigma_{2}\left(v_{R}\right)_{\mathrm{X}}\right. \\
& \left.-\epsilon_{\mathrm{XYZ}}\left(v_{R}\right)_{\mathrm{X}}^{T} \sigma_{2}\left(t_{R}\right)_{\mathrm{Y}} \sigma_{2}\left(v_{R}\right)_{\mathrm{Z}}+\left(v_{R}\right)_{\mathrm{X}}^{T} \sigma_{2}\left(t_{R}\right)_{\mathrm{X}} \sigma_{2} u_{R}-u_{R}^{T} \sigma_{2}\left(t_{R}\right)_{\mathrm{X}} \sigma_{2}\left(v_{R}\right)_{\mathrm{X}}\right\} .
\end{aligned}
$$


The last two terms in (3.26) render massive the $S U(2)_{L}$ triplets $s$ and $s_{R}$ as well as the doublets $\bar{q}$ and $v_{R}$. Once these heavy fields are integrated out, only six $S U(2)_{L}$ doublets in $q$ and $u$ remain. Below a scale $\Lambda_{L}$, the $S U(2)_{L}$ color force confines these fields into the meson matrix

$$
M_{L}=\left(\begin{array}{cc}
q_{i}^{\alpha}\left(\sigma_{2}\right)_{\alpha \beta} q_{j}^{\beta} & q_{i}^{\alpha}\left(\sigma_{2}\right)_{\alpha \beta} u_{R}^{\beta} \\
u_{R}^{\beta}\left(\sigma_{2}^{T}\right)_{\beta \alpha} q_{i}^{\alpha} & 0
\end{array}\right)
$$

and generates the additional superpotential term $W_{\text {dyn }}^{L}=\operatorname{Pf} M_{L} / \Lambda_{L}{ }^{4}$. After collecting together the separate quantum and classical contributions and renaming all composites in terms of the $N_{f}=5$ magnetic baryons

$$
\begin{aligned}
p_{i j} & =\epsilon_{\alpha \beta} q_{i}^{\alpha} q_{j}^{\beta} \\
r_{i} & =\epsilon_{\alpha \beta} \epsilon_{\dot{\alpha} \dot{\beta}} \epsilon_{I J} q_{i}^{\alpha} q_{I}^{\beta \dot{\beta}} t_{J}^{\dot{\alpha}}=-\epsilon_{\alpha \beta} q_{i}^{\alpha}\left(u_{R}\right)^{\beta} \\
t_{\mathrm{x}} & =\epsilon_{\alpha \beta} \epsilon_{\dot{\alpha} \dot{\beta}}\left(\sigma_{\mathrm{x}} \sigma_{2}\right)_{I J} q_{I}^{\alpha \dot{\alpha}} q_{J}^{\prime \beta \dot{\beta}}=i \epsilon_{\alpha \beta}\left(t_{R}\right)_{\mathrm{x}}^{\alpha \beta},
\end{aligned}
$$

we obtain the superpotential which controls the dynamics in the extreme infrared:

$$
\begin{aligned}
W_{\mathrm{tot}}= & W_{\mathrm{tree}}+W_{\mathrm{dyn}}^{L}+W_{\mathrm{dyn}}^{R} \\
\simeq & k t_{\mathrm{x}} t_{\mathrm{x}}+\epsilon_{\mathrm{xYz}} n_{\mathrm{x}}^{i} n_{\mathrm{Y}}^{j} p_{i j} t_{\mathrm{z}}+n_{\mathrm{x}}^{i} r_{i} t_{\mathrm{x}}+\epsilon^{i j k l m} p_{i j} p_{k l} r_{m} \\
& \quad+r_{i} m^{i j} r_{j}+k m^{i j} m^{k l} p_{i k} p_{j l}+m^{i j} n_{\mathrm{x}}^{k} n_{\mathrm{x}}^{l} p_{i k} p_{j l} .
\end{aligned}
$$

The functional form of this magnetic result coincides with the first seven terms in the electric superpotential in (2.8). As in all previous Pouliot-type duals, there are some remaining nonrenormalizable terms whose origin we have not been able to identify. But aside from these last four terms, we see that the magnetic theory properly reproduces the confining phase for the $N_{Q}=2 S O(10)$ model.

\section{The general dual}

We now consider the dual to the $S O(10)$ theory with arbitrary numbers of spinors and vectors. The enlarged electric theory has symmetry group

$$
G=S O(10)_{\text {local }} \times\left[S U\left(N_{f}\right) \times S U\left(N_{Q}\right) \times U(1)_{Y} \times U(1)_{R}\right]_{\text {global }}
$$

and superfield matter content 8

$$
\begin{aligned}
V_{\mu}^{i} & \sim\left(10 ; \square, 1 ;-2 N_{Q}, R\right) \\
Q_{I}^{A} & \sim\left(16 ; 1, \square ; N_{f}, R\right)
\end{aligned}
$$

8 To avoid excessive cluttering of composite operator indices, we label $S O(10)$ spinors with lower flavor indices even though they transform according to the fundamental irrep of $S U\left(N_{Q}\right)$. 
where $R=1-8 /\left(N_{f}+2 N_{Q}\right)$. Various operators act as gauge invariant coordinates on the moduli space of this new theory. We will focus upon those which generalize the $N_{Q}=2$ composites in (2.6):

$$
\begin{aligned}
K_{I J K L} & =Q_{(I}^{T} \Gamma^{\mu} C Q_{J} Q_{(K}^{T} \Gamma_{\mu} C Q_{L} \sim\left(1 ; 1, \text { 田; } 4 N_{f}, 4 R\right) \\
M^{(i j)} & =\left(V^{T}\right)^{i \mu} V_{\mu}^{j} \sim\left(1 ; \square, 1 ;-4 N_{Q}, 2 R\right) \\
N_{(I J)}^{i} & =Q_{(I}^{T} V^{i} C Q_{J} \sim\left(1 ; \square, \square ; 2 N_{f}-2 N_{Q}, 3 R\right) \\
P_{[I J]}^{[i j k]} & =\frac{1}{3 !} Q_{[I}^{T} V^{[i} V^{j} V^{k]} C Q_{J]} \sim\left(1 ; \text { 日, 日; } 2 N_{f}-6 N_{Q}, 5 R\right) \\
R_{I J K L}^{[i j k l]} & =\frac{1}{4 !} Q_{(I}^{T} \Gamma^{\mu} Q_{J} Q_{(K}^{T} \Gamma_{\mu} V^{[i} V^{j} V^{k} V^{l]} C Q_{L]} \sim\left(1 ; \text { 日, 田; } 4 N_{f}-8 N_{Q}, 8 R\right) \\
T_{(I J)}^{[i j k l m]} & =\frac{1}{5 !} Q_{(I}^{T} V^{[i} V^{j} V^{k} V^{l} V^{m]} C Q_{J} \sim\left(1 ; \text { 日, 口; } ; 2 N_{f}-10 N_{Q}, 7 R\right) .
\end{aligned}
$$

Determining the quantum numbers for the two-spinor operators $M, N, P$ and $T$ is straightforward. On the other hand, figuring out the $S U\left(N_{Q}\right)$ irrep assignments for the four-spinor hadrons $K$ and $R$ is not so trivial. Previously when we had only $N_{Q}=2$ spinor flavors, we used counting and anomaly arguments to deduce that these baryons were global $S U(2)$ singlets. Now that we have expanded the electric theory to include arbitrary numbers of spinors, neither the number of independent quartic spinor $S O(10)$ invariants nor their transformation rules under $S U\left(N_{Q}\right)$ are immediately obvious.

In order to address these questions, it is useful to recall two Fierz identities [44]:

$$
\begin{aligned}
& \left(\Gamma^{[\mu} \Gamma^{\nu} \Gamma^{\sigma} \Gamma^{\tau} \Gamma^{\lambda]} P_{ \pm}\right)_{A B}\left(\Gamma_{[\mu} \Gamma_{\nu} \Gamma_{\sigma} \Gamma_{\tau} \Gamma_{\lambda]} P_{ \pm}\right)_{C D}=0 \\
& \left(\Gamma^{[\mu} \Gamma^{\nu} \Gamma^{\lambda]} P_{ \pm}\right)_{A B}\left(\Gamma_{[\mu} \Gamma_{\nu} \Gamma_{\lambda]} P_{ \pm}\right)_{C D}=12\left(\Gamma^{\mu} P_{ \pm}\right)_{A B}\left(\Gamma^{\mu} P_{ \pm}\right)_{C D}+24\left(\Gamma^{\mu} P_{ \pm}\right)_{A D}\left(\Gamma^{\mu} P_{ \pm}\right)_{C B} .
\end{aligned}
$$

The first relation implies that the $S O(10)$ invariant $Q_{\left({ }_{I}\right.} \Gamma^{[\mu \nu \sigma \tau \lambda]} C Q_{J)} Q_{\left({ }_{K}\right.} \Gamma_{[\mu \nu \sigma \tau \lambda]} C Q_{L)}$ simply vanishes. The second guarantees that the operator $Q_{\left[{ }_{I}\right.} \Gamma^{[\mu \nu \lambda]} C Q_{J]} Q_{\left[{ }_{K}\right.} \Gamma_{[\mu \nu \lambda]} C Q_{L]}$ can be decomposed in terms of others of the form $Q_{(I} \Gamma^{\mu} C Q_{J} Q_{(K} \Gamma_{\mu} C Q_{L)}$. So without loss of generality, we need only consider four-spinor composites of this last type. Such hadrons transform under $S U\left(N_{Q}\right)$ according to the product representation

$$
(\square \times \square)_{S}=\square+\boxplus \text {. }
$$

Since the $S O(10)$ theory with just one spinor has no flat directions 455, holomorphic invariants associated with the totally symmetric irrep שח must vanish. We thus deduce that four-spinor operators transform only according to the "window frame" irrep $\boxplus$. 
The expanded $S O(10)$ model is infrared free when its Wilsonian beta function coefficient $b_{0}=24-N_{f}-2 N_{Q}$ is nonpositive. In contrast, the theories with $N_{Q}=1,2,3$ and 4 spinors confine provided they respectively possess $N_{f} \leq 6,5,3$ and 1 vectors. In between these two limits, the $S O(10)$ model is asymptotically free, but gauge singlet operators do not suffice to describe its infrared behavior. Instead, the physics of this last phase can be described in terms of a dual which naturally generalizes our earlier $S U\left(N_{f}-3\right) \times S p(2)$ product theory.

The magnetic dual is based upon the symmetry group

$$
\widetilde{G}=\left[S U\left(\tilde{N}_{c}\right) \times S p\left(2 \tilde{N}_{c}^{\prime}\right)\right]_{\text {local }} \times\left[S U\left(N_{f}\right) \times S U(2) \times U(1)_{Y} \times U(1)_{R}\right]_{\text {global }}
$$

where $\tilde{N}_{c}=N_{f}+2 N_{Q}-7$ and $\tilde{N}_{c}^{\prime}=N_{Q}-1$. It is important to note that only an $S U(2)$ subgroup of the global $S U\left(N_{Q}\right)$ which rotates the spinors in the electric theory is realized in the ultraviolet on the magnetic side. The $S U(2)$ subgroup is embedded inside $S U\left(N_{Q}\right)$ such that the fundamental $N_{Q}$-dimensional irrep of the latter maps onto the $N_{Q}$-dimensional irrep of the former. The full $S U\left(N_{Q}\right)$ global symmetry is realized in the magnetic theory only at long distances.

The new dual's matter content generalizes that which we previously found in (3.22):

$$
\begin{aligned}
q_{i}^{\alpha} & \sim\left(\square, 1 ; \bar{\square}, 1 ; Y_{q}, R_{q}\right) \\
{q_{I}^{\prime \alpha}}_{I}^{\alpha \dot{\alpha}} & \sim\left(\square, \square ; 1,2 ; Y_{q^{\prime}}, R_{q^{\prime}}\right) \\
\bar{q}_{\alpha\left(I_{1} \cdots I_{2 N_{Q}}-2\right)} & \sim\left(\bar{\square}, 1 ; 1,2 N_{Q}-1 ; Y_{\bar{q}}, R_{\bar{q}}\right) \\
s_{\alpha \beta} & \sim\left(\bar{\square}, 1 ; 1,1 ; Y_{s}, R_{s}\right) \\
t_{\left(I_{1} \cdots I_{2} N_{Q}-3\right)}^{\dot{\alpha}} & \sim\left(1, \square ; 1,2 N_{Q}-2 ; Y_{t}, R_{t}\right) . \\
m^{(i j)} & \sim\left(1,1 ; \square, 1 ; Y_{m}, R_{m}\right) \\
n_{\left(I_{1} \cdots I_{2} N_{Q^{-2}}\right)}^{i} & \sim\left(1,1 ; \square, 2 N_{Q}-1 ; Y_{n}, R_{n}\right) .
\end{aligned}
$$


The hypercharge and R-charge assignments for these fields are listed below:

$$
\begin{aligned}
Y_{q} & =\frac{2}{\tilde{N}_{c}}\left(N_{Q} \tilde{N}_{c}-N_{f} \tilde{N}_{c}^{\prime}\right) & R_{q} & =\frac{9-2 N_{Q}}{\tilde{N}_{c}} R \\
Y_{q^{\prime}} & =-2 N_{f} \frac{\tilde{N}_{c}^{\prime}}{\tilde{N}_{c}} & R_{q^{\prime}} & =\frac{N_{f}+2}{\tilde{N}_{c}} R \\
Y_{\bar{q}} & =-2 N_{f} \frac{\tilde{N}_{c}-\tilde{N}_{c}^{\prime}}{\tilde{N}_{c}} & R_{\bar{q}} & =-\frac{N_{f}^{2}+2 N_{f} N_{Q}-22 N_{f}-28 N_{Q}+96}{\tilde{N}_{c}\left(N_{f}+2 N_{Q}\right)} \\
Y_{s} & =4 N_{f} \frac{\tilde{N}_{c}^{\prime}}{\tilde{N}_{c}} & R_{s} & =2 \frac{4 N_{Q}^{2}+2 N_{f} N_{Q}-N_{f}-18 N_{Q}+16}{\tilde{N}_{c}\left(N_{f}+2 N_{Q}\right)} \\
Y_{t} & =2 N_{f} & R_{t} & =2 R . \\
Y_{m} & =-4 N_{Q} & R_{m} & =2 R \\
Y_{n} & =2 N_{f}-2 N_{Q} & R_{n} & =3 R .
\end{aligned}
$$

The tree level magnetic superpotential similarly extends (3.12) in the $N_{Q}=2$ model:

$$
\begin{aligned}
& W_{\mathrm{mag}}=\frac{1}{\mu_{1}^{2}} m^{(i j)} q_{i}^{\alpha} s_{\alpha \beta} q_{j}^{\beta}+\lambda_{1} \epsilon^{I J} J_{\dot{\alpha} \dot{\beta}} q_{I}^{\prime \alpha \dot{\alpha}} s_{\alpha \beta} q_{J}^{\prime \beta \dot{\beta}} \\
& +\frac{1}{\mu_{2}^{2}} \epsilon^{I_{1} J_{1}} \cdots \epsilon^{I_{2 N_{Q}-2 J_{2 N} Q^{-2}}} q_{i}^{\alpha} n_{\left(I_{1} \cdots I_{2 N} N_{Q}-2\right)}^{i} \bar{q}_{\alpha\left(J_{1} \cdots J_{2 N_{Q}-2}\right)}
\end{aligned}
$$

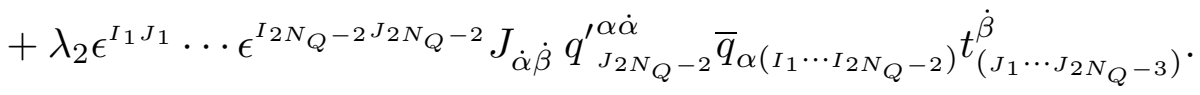

Here $J_{\dot{\alpha} \dot{\beta}}=\left(1_{\tilde{N}_{c}^{\prime} \times \tilde{N}_{c}^{\prime}} \otimes i \sigma_{2}\right)_{\dot{\alpha} \dot{\beta}}$ denotes the antisymmetric metric that remains invariant under $\operatorname{Sp}\left(2 \tilde{N}_{c}^{\prime}\right)$ rotations. The coefficients $\mu_{1,2}$ and $\lambda_{1,2}$ represent dimensionful and dimensionless coupling constants which we again set to unity for simplicity.

Several points about this new product dual should be noted. Firstly, it satisfies all necessary anomaly checks. The $S U\left(\tilde{N}_{c}\right)^{3}, S U\left(\tilde{N}_{c}\right)^{2} U(1)_{Y}, S U\left(\tilde{N}_{c}\right)^{2} U(1)_{R}, S p\left(2 \tilde{N}_{c}^{\prime}\right)^{2} U(1)_{Y}$, and $S p\left(2 \tilde{N}_{c}^{\prime}\right)^{2} U(1)_{R}$ anomalies vanish, and an even number of fundamentals transform under the $S p\left(2 \tilde{N}_{c}^{\prime}\right)$ gauge group. All anomaly matching conditions associated with the common $S U\left(N_{f}\right) \times S U(2) \times U(1)_{Y} \times U(1)_{R}$ global symmetry group are also satisfied. We display these 't Hooft anomaly values in Table 3. Secondly, we observe from the Wilsonian beta functions coefficients ${\tilde{b_{0}}}^{S U\left(\tilde{N}_{c}\right)}=2 N_{f}+2 N_{Q}-16$ and ${\tilde{b_{0}}}^{S p\left(2 \tilde{N}_{c}^{\prime}\right)}=8-N_{f}$ that the dual never resides within a free magnetic phase for any values of $N_{f}$ and $N_{Q}$. We also note that the $N_{Q}=3\left(N_{Q}=4\right)$ magnetic theory enters into the confining phase when $N_{f}=3$ $\left(N_{f}=1\right)$ in a fashion similar to that for the $N_{Q}=2$ model. Finally, the symplectic gauge group in (4.6) sees only fundamental irrep matter. By dualizing the $S p\left(2 \tilde{N}_{c}^{\prime}\right)$ color factor, 


\begin{tabular}{|c|c|}
\hline Global Anomaly & Value \\
\hline$S U\left(N_{f}\right)^{3}$ & 10 \\
$S U\left(N_{f}\right)^{2} U(1)_{Y}$ & $-20 N_{Q}$ \\
$S U\left(N_{f}\right)^{2} U(1)_{R}$ & $-\frac{80}{N_{f}+2 N_{Q}}$ \\
$S U(2)^{2} U(1)_{Y}$ & $\frac{8}{3} N_{f} N_{Q}\left(N_{Q}{ }^{2}-1\right)$ \\
$S U(2)^{2} U(1)_{R}$ & $\frac{64 N_{Q}\left(1-N_{Q}{ }^{2}\right)}{3\left(N_{f}+2 N_{Q}\right)}$ \\
$U(1)_{Y}$ & $-4 N_{f} N_{Q}$ \\
$U(1)_{Y}^{3}$ & $16 N_{f} N_{Q}\left(N_{f}^{2}-5 N_{Q}{ }^{2}\right)$ \\
$U(1)_{R}$ & $-\frac{38 N_{Q}+35 N_{f}}{N_{f}+2 N_{Q}}$ \\
$U(1)_{R}^{3}$ & $\frac{\text { numer }}{\left(N_{f}+2 N_{Q}\right)^{3}}$ \\
$U(1)_{Y}^{2} U(1)_{R}$ & $-64 N_{f} N_{Q} \frac{2 N_{f}+5 N_{Q}}{N_{f}+2 N_{Q}}$ \\
$U(1)_{R}^{2} U(1)_{Y}$ & $-256 \frac{N_{f} N_{Q}}{\left(N_{f}+2 N_{Q}\right)^{2}}$ \\
\hline
\end{tabular}

Table 3: Global 't Hooft anomalies in the $S O(10)$ theory with $N_{f}$ vectors and $N_{Q}$ spinors and its $S U\left(\tilde{N}_{c}\right) \times S p\left(2 \tilde{N}_{c}^{\prime}\right)$ dual. The numerator of the $U(1)_{R}^{3}$ anomaly equals $360 N_{Q}{ }^{3}+45 N_{f}^{3}+540 N_{Q}^{2} N_{f}+270 N_{f}^{2} N_{Q}-8192 N_{Q}-5120 N_{f}$.

we can derive another magnetic counterpart to the general $S O(10)$ model [1, 46]. The symmetric tensor disappears from the resulting product dual, and three antisymmetric fields take its place. We outline the basic structure of this second dual in Appendix B. As it is more complicated but not more illuminating than the magnetic theory we have already discussed, we will not consider it further.

As an additional check, we consider flows induced by mass deformations. If we add a tree level superpotential $W=m V^{N_{f}} V^{N_{f}}$ which gives mass to a vector in the $S O(10)$ theory, we find that the magnetic $S U\left(N_{f}-2 N_{Q}-7\right)$ color group properly breaks down to $S U\left(\left[N_{f}-1\right]-2 N_{Q}-7\right)$. On the other hand, we cannot give mass to any of the 
$S O(10)$ spinors. Instead, we may Higgs $S O(10)$ down to $S O(9)$ along the flat direction with nonzero $\left\langle V^{N_{f}}\right\rangle$, and then add a mass term $W=\left\langle V^{N_{f}}\right\rangle Q_{N_{Q}} Q_{N_{Q}}$ which removes one spinor. The effect on the magnetic theory is quite complicated. A long and careful analysis demonstrates that the gauge group is properly reduced and the correct matter fields are removed. In the case when $N_{Q}=2$, the $S U\left(N_{f}-3\right) \times S p(2)$ gauge group breaks to $S U\left(N_{f}-5\right)$, and instanton effects in the broken $S p(2)$ generate a det $s$ term in the magnetic superpotential. The resulting theory thus reproduces the dual counterpart of $S O(9)$ with one spinor, which follows from the dual to the $N_{Q}=1 S O(10)$ model [3, 30]. Since all earlier Pouliot-type duals can be obtained from the $N_{Q}=1$ theory, our duality transformation contains these results as special cases.

We next examine the mapping of gauge invariant operators between the electric and magnetic theories. This issue is complicated by the partial $S U(2)$ realization of the $S U\left(N_{Q}\right)$ global symmetry in the dual. In order to match operators, we need to first decompose the electric hadrons' $S U\left(N_{Q}\right)$ representations under the $S U(2)$ subgroup following the embedding $\square \rightarrow N_{Q}$. For example, symmetric and antisymmetric tensors of $S U\left(N_{Q}\right)$ break apart as

$$
\begin{aligned}
& \square \rightarrow \sum_{i=0}^{i_{\max }}\left(2 N_{Q}-1-4 i\right) \quad \text { where } i_{\max }= \begin{cases}\left(N_{Q}-1\right) / 2 & N_{Q} \text { odd } \\
\left(N_{Q}-2\right) / 2 & N_{Q} \text { even }\end{cases} \\
& \text { 日 } \rightarrow \sum_{i=0}^{i_{\max }}\left(2 N_{Q}-3-4 i\right) \quad \text { where } i_{\max }= \begin{cases}\left(N_{Q}-3\right) / 2 & N_{Q} \text { odd } \\
\left(N_{Q}-2\right) / 2 & N_{Q} \text { even. }\end{cases}
\end{aligned}
$$

Combining this information with abelian charge assignments, we readily find that the dual baryons

$$
\begin{aligned}
& p=q^{N_{f}-3} q^{\prime 2 N_{Q}-4} \\
& t=q^{N_{f}-5} q^{\prime 2 N_{Q}-2}
\end{aligned}
$$

match onto $P$ and $T$ in (4.3). Similarly, the elementary $n$ partons in (4.7) along with the magnetic composites

$$
n^{\prime}=q^{N_{f}-1} q^{\prime 2 N_{Q}-6}
$$

account for all the $N$ fields in the electric theory.

Whereas mapping two-spinor $S O(10)$ operators is straightforward, finding dual counterparts to four-spinor hadrons is much more involved. We will concentrate upon identifying the magnetic partners to the operator $K$ in (4.3). First, we decompose its $S U\left(N_{Q}\right)$ $\boxplus$ irrep under the $S U(2)$ subgroup. The results for small values of $N_{Q}$ are displayed 
in Table 4. We next observe that the composites $t^{2}, q^{N_{f}} q^{\prime \tilde{N}_{c}-N_{f}} t$ and $\left(q^{N_{f}} q^{\prime \tilde{N}_{c}-N_{f}}\right)^{2}$ have the same hypercharge and R-charge as $K$. The total number of these magnetic hadrons naively appears to exceed $\operatorname{dim}(\boxplus)=N_{Q}{ }^{2}\left(N_{Q}{ }^{2}-1\right) / 12$. But it is important to remember that we should only count operators which are not set to zero by equations of motion. After carefully considering the effect of each term in $W_{\text {mag }}$, we find that the numbers of such nonredundant $t^{2}, q^{N_{f}} q^{\prime \tilde{N}_{c}-N_{f}} t$ and $\left(q^{N_{f}} q^{\prime \tilde{N}_{c}-N_{f}}\right)^{2}$ baryons respectively equal $\left(2 N_{Q}-3\right)\left(N_{Q}-1\right),\left(2 N_{Q}-3\right)\left(N_{Q}-2\right)\left(N_{Q}-3\right) / 2$ and $\left(N_{Q}-2\right)\left(N_{Q}-3\right)^{2}\left(N_{Q}-4\right) / 12$. As Table 4 illustrates, these magnetic composites precisely account for all components of $K$ in the electric theory.

\begin{tabular}{|c|c|c|c|c|}
\hline$N_{Q}$ & $K$ & $t^{2}$ & $q^{N_{f}} q^{\prime \tilde{N}_{c}-N_{f}} t$ & $\left(q^{N_{f}} q^{\prime \tilde{N}_{c}-N_{f}}\right)^{2}$ \\
\hline 2 & $1=(1)$ & 1 & 0 & 0 \\
\hline 3 & $6=(5)+(1)$ & 6 & 0 & 0 \\
\hline 4 & $20=(9)+2(5)+(1)$ & 15 & 5 & 0 \\
\hline 5 & $50=(13)+2(9)+(7)+2(5)+2(1)$ & 28 & 21 & 1 \\
\hline
\end{tabular}

Table 4: Mapping of the electric theory four-spinor composite $K$ onto nonredundant magnetic baryons. The $S U(2)$ decomposition of the $S U\left(N_{Q}\right) \boxplus$ irrep is displayed in the second column.

The final check of the duality transformation which we perform involves an intricate renormalization group flow analysis that generalizes the procedure introduced in ref. [27]. We first deconfine the magnetic theory's symmetric tensor and then consider the resulting $S O\left(\tilde{N}_{c}+4\right) \times S U\left(\tilde{N}_{c}\right) \times S p\left(2 \tilde{N}_{c}^{\prime}\right)$ model. We investigate flows in this theory for large and small values of the ratio $\Lambda_{S O} / \Lambda_{S U}$, where $\Lambda_{S O}\left[\Lambda_{S U}\right]$ denotes the strong coupling scale for $S O\left(\tilde{N}_{c}+4\right)\left[S U\left(\tilde{N}_{c}\right)\right]$. Since holomorphy ensures that phase transitions between these two regions cannot occur [35], the low energy physics of both must be qualitatively similar. After utilizing several different duality transformations, we find that the triple product theory flows to the same $S O(10)$ fixed point for both large and small values of $\Lambda_{S O} / \Lambda_{S U}$. This demonstrates that our dual is consistent with Seiberg's well-known results.

We first consider the $\Lambda_{S O} \gg \Lambda_{S U}$ case. The $S O\left(\tilde{N}_{c}+4\right)$ factor then grows strongly coupled, and the renormalization group flow passes through the intermediate stages 


$$
\begin{gathered}
S O\left(N_{f}+2 N_{Q}-3\right) \times S U\left(N_{f}+2 N_{Q}-7\right) \times S p\left(2 N_{Q}-2\right) \\
\downarrow(\mathrm{SO} \text { confinement }) \\
S U\left(N_{f}+2 N_{Q}-7\right) \times S p\left(2 N_{Q}-2\right) \\
\downarrow(\mathrm{SU} \times \text { Sp duality }) \\
S O(10)
\end{gathered}
$$

before arriving at the final infrared fixed point. On the other hand, the flow pattern takes the form

$$
\begin{gathered}
S O\left(N_{f}+2 N_{Q}-3\right) \times S U\left(N_{f}+2 N_{Q}-7\right) \times S p\left(2 N_{Q}-2\right) \\
\downarrow(\text { SU duality }) \\
\qquad O\left(N_{f}+2 N_{Q}-3\right) \times S U\left(2 N_{Q}+3\right) \times S p\left(2 N_{Q}-2\right) \\
\downarrow(\text { SO duality }) \\
\qquad O(10) \times S U\left(2 N_{Q}+3\right) \times S p\left(2 N_{Q}-2\right) \\
\downarrow(\text { SU } \times \text { Sp duality }) \\
\qquad O(10) \times S O(10) \\
\downarrow(\text { Tree level breaking }) \\
S O(10)
\end{gathered}
$$

when $\Lambda_{S U} \gg \Lambda_{S O}$. The first two steps involve Seiberg's duality transformations [1, 20], while the third utilizes the variant of our duality transformation discussed in Appendix C. The closure of the two duality chains in (4.13) and (4.14) constitutes a highly nontrivial consistency check on our results.

The details underlying this flow analysis are presented in Appendix D. The interested reader will find them remarkable in their complexity and intricacy. 


\section{A composite Standard Model}

In his original work on $\mathcal{N}=1$ duality, Seiberg speculated that the Standard Model might represent a low energy effective description of a more fundamental theory with a totally different gauge group [1]. In such a scenario, some or all of the ordinary matter and gauge particles would be composite. As the Minimal Supersymmetric Standard Model is weakly coupled at all energies between the SUSY breaking and Planck scales, there is little point in constructing a dual to it. But if Nature contains additional vectorlike matter with masses between $\Lambda_{\text {SUSY }}$ and $\Lambda_{\text {Planck }}$, the Standard Model with these extra fields could become strongly interacting at high energies. In this case, it would clearly be beneficial to find a weakly coupled description of the microscopic physics.

If one simply adds matter charged in fundamental representations and tries to apply Seiberg's $S U(N)$ duality transformation to the individual nonabelian factors of the Standard Model gauge group, one encounters an unending sequence of duals involving ever larger gauge groups and shorter energy regimes [47]. Some attempts to evade this "Duality Wall" have been made in the past [48]. But to properly overcome this problem, one needs either to dualize two or three of the Standard Model's subgroups simultaneously or to unify the Standard Model within a larger group. We now present a toy model which follows the second approach.

We start with an $S O(10)$ grand unified theory. In order to break $S O(10)$ down to $S U(3) \times S U(2) \times U(1)$, we need a scalar field in the adjoint representation. Since we do not yet know a simple dual to an $S O(10)$ theory with spinor, vector and adjoint matter, we adopt the deconfinement method to build the adjoint [21]. $S p\left(2 N_{c}\right)$ theories with $2 N_{c}+4$ fields in the fundamental representation confine and are described at low energy in terms of mesons antisymmetric in flavor. Consequently, if we take an $S O(10)$ theory with $N_{f}$ vectors and gauge an $S p(6)$ subgroup of its $S U\left(N_{f}\right)$ flavor symmetry, we can generate an adjoint of $S O(10)$ below the $S p(6)$ confining scale.

We are thus motivated to consider a product theory with nonabelian symmetry group $[S O(10) \times S p(6)]_{\text {local }} \times\left[S U\left(N_{f}\right) \times S U(3)\right]_{\text {global }}$ and matter content $V_{\mu}^{i} \sim\left(10,1 ; N_{f}, 1\right)$, $Z_{\mu}^{\dot{\alpha}} \sim(10,6 ; 1,1), Q_{I}^{A} \sim(16,1 ; 1,3)$. The $S O(10)$ factor is free in the infrared provided $N_{f} \geq 12$. On the other hand, the $S p(6)$ gauge group grows strong at energies below its strong coupling scale $\Lambda_{S P}$. It confines the $Z$ partons into the antisymmetric mesons $A_{[\mu \nu]}=Z_{\mu}^{\dot{\alpha}} J_{\dot{\alpha} \dot{\beta}} Z_{\nu}^{\dot{\beta}}$ and dynamically generates the superpotential $W_{\mathrm{dyn}}=\operatorname{Pf} A / \Lambda_{S P}^{7}$. When this quantum effect is combined with an appropriate classical superpotential $W=f(A)$, 
the $S O(10)$ gauge group breaks to $S U(3)_{C} \times S U(2)_{L} \times U(1)_{Y} \times U(1)_{B-L}$. The resulting low energy theory then possesses the Standard Model gauge group and chiral matter content along with extra vectorlike matter fields. These ingredients are precisely those we need to investigate Seiberg's suggestion.

Using the results of section 4 , we regard this $S O(10) \times S p(6)$ theory itself as the low energy limit of an $S U\left(N_{f}+5\right) \times S p(4) \times S p(6)$ model, provided $N_{f} \geq 12$. The $S p(6)$ factor acts as a weakly coupled spectator under the $S O(10) \leftrightarrow S U\left(N_{f}-5\right) \times S p(4)$ duality transformation.

The principle features of our toy model are thus the following. At the Planck or string scale, it is based upon an $S U\left(N_{f}+5\right) \times S p(4) \times S p(6)$ gauge group. This theory flows down to an $S O(10) \times S p(6)$ model. The $S O(10)$ factor is infrared free for $N_{f} \geq 12$, while the $S p(6)$ force grows strong at low energies and confines six vectors into an $S O(10)$ adjoint. A tree level superpotential can induce an expectation value for the adjoint field which breaks the intermediate grand unified theory down to the Standard Model. Since the adjoint and extra vectors are nonchiral, we can arrange for unwanted components of these fields to develop large masses. We are then left with three generations of Standard Model families at low energies. Additional terms in the classical superpotential may be added to provide Yukawa couplings to these matter fields.

This model is certainly contrived. However, it has a sensible high energy description and flows to the Standard Model at low energies as a consequence of strong coupling effects. If some scenario like this actually operates in the real world, all Standard Model gauge bosons and matter fields could indeed represent composite low energy degrees of freedom.

\section{Conclusion}

The duals which we have constructed in this article exhibit a number of novel features. Firstly, our transformation represents an essentially new type of product group duality. It does not follow from Seiberg's results, and it is not related by confinement to other known dual pairs. Secondly, it provides dual descriptions of supersymmetric theories with arbitrary numbers of two distinct types of matter. All previous electric theories for which magnetic duals have been found involve at most one infinite chain of matter fields. As can be seen in fig. 1, counterparts to numerous other theories may be derived along various spinor and vector flat directions. Thirdly, the role which accidental symmetries plays in these theories is unusual. The $S O(10)$ model with $N_{f}$ vectors and $N_{Q}$ spinors possesses an 
$S U\left(N_{Q}\right)$ global symmetry. Only an $S U(2)$ subgroup, under which the spinors transform

according to the $N_{Q}$-dimensional representation, is manifest in the $S U\left(\tilde{N}_{c}\right) \times S p\left(2 \tilde{N}_{c}^{\prime}\right)$ dual. As a result, the size of the global subgroup present in the classical dual does not depend upon $N_{Q}$. Instead, the $N_{Q}$ dependence resides in the matter representations of the dual theory. Finally, $S O(10)$ models with spinors have clear implications for particle physics. We have constructed a toy model with a completely unfamiliar gauge group and matter content which flows down to the Standard Model via a three-generation $S O(10)$ grand unified theory. This scenario realizes Seiberg's suggestion that all Standard Model gauge bosons and matter fields might represent low energy effective degrees of freedom of some more fundamental gauge theory.

\section{Acknowledgments}

We thank Howard Georgi for helpful discussions. We also acknowledge support from DOE Grant DE-FG02-96ER40559 (MB), the National Science Foundation under Grant \#PHY-9218167 (PC), DOE grant DE-FG03-92-ER40701 and the DuBridge Fellowship Foundation (PK), and the National Science Foundation under Grant PHY-9513835 and the WM Keck Foundation (MJS).

\section{Appendix A. Mapping $Q^{4} W^{2}$}

In $S O\left(N_{c}\right)$ dual pairs, gauge invariant operators involving the field strength $W$ play an important role in the matching of chiral rings [20]. We have already seen such composites in (3.6) - (3.9). One can construct many more of this type. In this appendix, we examine the mapping of primary $Q^{4} W^{2}$ operators in the $N_{Q}=2 S O(10)$ model. We adopt the gauge conventions of refs. [49 51] and let $\mathcal{D}$ denote a super convariant derivative which includes the gauge connection.

$W^{2}=\epsilon^{a b} W_{a} W_{b}$ transforms under $S O(10)$ according to the symmetric product $(45 \times$ $45)_{S}=1+54+210+770$. We recall from (2.5) that $Q^{2}$ transforms as $16 \times 16=10_{S}+$ $120_{A}+126_{S}$. The relevant $Q^{4}$ products we need to consider in order to form $S O(10)$ invariants are thus $10 \times 10=54+\ldots, 10 \times 120=210+\ldots, 10 \times 126=210+\ldots, 120 \times 120=$ $54+210+\ldots, 120 \times 126=210+\ldots$ and $126 \times 126=54+\ldots$. Most of the possible $Q^{4} W^{2}$ 
combinations are either not primary or else not independent. For example, the $10 \times 10$ operator $\epsilon_{a b} Q \Gamma^{\mu} Q Q \Gamma^{\nu} Q W_{\mu \sigma}^{a} W_{\nu \sigma}^{b}$ is a descendant as $W_{\sigma \nu}^{b} Q \Gamma^{\nu} Q \propto \overline{\mathcal{D}}_{\dot{a}} \overline{\mathcal{D}}^{\dot{a}} \mathcal{D}^{b} Q \Gamma_{\sigma} Q$ [52]. Similarly, $Q \Gamma^{\left[\mu_{1} . . \mu_{l}\right]} \Gamma^{\left[\nu_{1} \nu_{2}\right]} Q W_{\nu_{1} \nu_{2}}$ is a descendant since $W_{\nu_{1} \nu_{2}}^{b} \Gamma^{\nu_{1} \nu_{2}} Q=\overline{\mathcal{D}}_{\dot{a}} \overline{\mathcal{D}}^{\dot{a}} \mathcal{D}^{b} Q$. As a result, $Q^{4}$ products arising from $10 \times 120$ yield only descendant $Q^{4} W^{2}$ operators.

After a systematic search, we find just two independent primaries which come from the $120 \times 120$ product:

$$
\begin{aligned}
& U=\epsilon_{a b} Q_{1} \Gamma^{\left[\mu_{1} \mu_{2} \mu_{3}\right]} Q_{2} Q_{1} \Gamma^{\left[\mu_{1} \nu_{2} \nu_{3}\right]} Q_{2} W_{\mu_{2} \nu_{2}}^{a} W_{\mu_{3} \nu_{3}}^{b} \\
& V=\epsilon_{a b} Q_{1} \Gamma^{\left[\mu_{1} \mu_{2} \mu_{3}\right]} Q_{2} Q_{1} \Gamma^{\left[\mu_{1} \mu_{2} \nu_{3}\right]} Q_{2} W_{\mu_{3} \sigma}^{a} W_{\nu_{3} \sigma}^{b} .
\end{aligned}
$$

Both of these operators are singlets under the global $S U(2)$ which rotates the two spinors in the electric theory. On the magnetic side,

$$
\begin{aligned}
& u=\operatorname{det} s \\
& v=\epsilon_{\dot{\alpha} \dot{\beta}} \epsilon_{\dot{\gamma} \dot{\delta}} \epsilon^{I J} \epsilon^{K L} q_{I}^{\prime \alpha \dot{\alpha}} t_{J}^{\dot{\beta}} s_{\alpha \beta} q_{K}^{\prime \beta \dot{\gamma}} t_{L}^{\dot{\delta}}
\end{aligned}
$$

share precisely the same quantum numbers as $U$ and $V$. We thus identify the electric operators with linear combinations of the magnetic operators.

\section{Appendix B. A second general dual}

It is possible to construct another dual to the $S O(10)$ model with $N_{f}$ vectors and $N_{Q}$ spinors which differs from the $S U\left(\tilde{N}_{c}\right) \times S p\left(2 \tilde{N}_{c}^{\prime}\right)$ theory discussed in section 4 . We observe that our original dual reduces to a symplectic theory with only fundamental and singlet matter fields when the $S U\left(N_{f}\right)$ gauge coupling is set to zero. It is then straightforward to apply Seiberg's duality to the $S p\left(2 \tilde{N}_{c}^{\prime}\right)$ gauge group [1,46]. After replacing the $S p\left(2 \tilde{N}_{c}^{\prime}\right)$ theory with its dual and restoring the $S U\left(\tilde{N}_{c}\right)$ gauge coupling, we find a new magnetic description of the $S O(10)$ model. We sketch a derivation of this second dual below.

We start with the magnetic theory of section 4 . When the $S U\left(\tilde{N}_{c}\right)$ gauge coupling is turned off, the fields $q_{I}^{\alpha \dot{\alpha}}$ and $t_{\dot{I}}^{\dot{\alpha}}$ in (4.7) become $2 \tilde{N}_{c}$ and $2 N_{Q}-2$ fundamentals under the remaining $S p\left(2 \tilde{N}_{c}^{\prime}\right)$ group. 9 We recall that the dual to $S p\left(2 N_{c}\right)$ with $N_{f}$ fundamentals has gauge group $S p\left(N_{f}-2 N_{c}-4\right)$. The gauge group in our new magnetic theory is thus $S U\left(N_{f}+2 N_{Q}-7\right) \times S p\left(2 N_{f}+4 N_{Q}-18\right)$.

9 In this appendix, we adopt the index $\dot{I}$ as shorthand for $\left(I_{1} \cdots I_{2 N_{Q}-3}\right)$. 
Seiberg's duality transformation introduces certain mesons as fundamental fields on the magnetic side. These mesons correspond to $S p\left(2 \tilde{N}_{c}^{\prime}\right)$ invariant combinations of $q_{I}^{\prime \alpha \dot{\alpha}}$ and $t_{\dot{\bar{\alpha}}}^{\dot{\alpha}}$ :

$$
\begin{aligned}
\left(m^{(a)}\right)_{(I J)}^{[\alpha \beta]} & \sim q_{(I}^{\prime \alpha \dot{\alpha}} q_{J J}^{\prime \beta \dot{\beta}} J_{\dot{\alpha} \dot{\beta}} & \left(m^{(s)}\right)^{(\alpha \beta)} & \sim q_{1}^{\prime \alpha \dot{\alpha}} q_{2}^{\prime \beta \dot{\beta}} J_{\dot{\alpha} \dot{\beta}} \\
\left(m_{t}\right)_{[\dot{I} \dot{J}]} & \sim t_{\dot{I}}^{\dot{\alpha}} t_{\dot{J}}^{\dot{\beta}} J_{\dot{\alpha} \dot{\beta}} & \left(m_{q^{\prime} t}\right)_{I \dot{J}}^{\alpha} & \sim q_{I}^{\prime \alpha \dot{\alpha}} t_{\dot{J}}^{\dot{\beta}} J_{\dot{\alpha} \dot{\beta}} .
\end{aligned}
$$

It should be noted that $\left(m_{q^{\prime} t}\right)_{I J}^{\alpha}$ is not irreducible under the $S U(2)$ flavor group, but is rather the direct sum of two irreps with dimensions $2 N_{Q}-1$ and $2 N_{Q}-3$. When expressed in terms of the mesons, the magnetic superpotential of (4.9) becomes

$$
W_{\mathrm{mag}}=m q s q+m^{(s)} s+q n \bar{q}+m_{q^{\prime}} \bar{q} .
$$

We must also include superpotential couplings between the dual quarks $\hat{q}_{\alpha I}^{\prime \dot{\alpha}}$ and $\hat{t}_{\dot{I}}^{\dot{\alpha}}$ and the fields in (B.1):

$$
\Delta W_{\mathrm{mag}}=m^{(s)} \hat{q}^{\prime} \hat{q}^{\prime}+m^{(a)} \hat{q}^{\prime} \hat{q}^{\prime}+m_{q^{\prime} t} \hat{q}^{\prime} \hat{t}+m_{t} \hat{t} \hat{t} .
$$

The bilinear terms in (B.2) give mass to $m^{(s)}, s, \bar{q}$ and $2 N_{Q}-1$ components of $m_{q^{\prime} t}$. Upon integrating out these heavy fields, we arrive at the new dual theory which has nonabelian symmetry group 10

$$
\widetilde{G}^{\prime}=\left[S U\left(N_{f}+2 N_{Q}-7\right) \times S p\left(2 N_{f}+4 N_{Q}-18\right)\right]_{\text {local }} \times\left[S U\left(N_{f}\right) \times S U(2)\right]_{\text {global }},
$$

matter content

$$
\begin{aligned}
q & \sim(\square, 1 ; \bar{\square}, 1) \\
\tilde{q}^{\prime} & \sim(\bar{\square}, \square ; 1,2) \\
\hat{t} & \sim\left(1, \square ; 1,2 N_{Q}-2\right) \\
m^{(a)} & \sim(\boxminus, 1 ; 1,3) \\
m_{q^{\prime} t} & \sim\left(\square, 1 ; 1,2 N_{Q}-3\right) \\
m & \sim(1,1 ; \square, 1) \\
n & \sim\left(1,1 ; \square, 2 N_{Q}-1\right) \\
m_{t} & \sim\left(1,1 ; 1,\left[\left(2 N_{Q}-2\right) \times\left(2 N_{Q}-2\right)\right]_{A}\right)
\end{aligned}
$$

and tree level superpotential

$$
W_{\mathrm{mag}}+\Delta W_{\mathrm{mag}}=m q q \hat{q}^{\prime} \hat{q}^{\prime}+q n \hat{q}^{\prime} \hat{t}+m^{(a)} \hat{q}^{\prime} \hat{q}^{\prime}+m_{q^{\prime} t} \tilde{q}^{\prime} t+m_{t} \hat{t} \hat{t} .
$$

10 For brevity's sake, we do not display any $U(1)$ charge assignments in appendices B, C or D. 
As in the original dual, the Wilsonian beta function coefficients for the new magnetic gauge couplings

$$
\begin{aligned}
b_{0}^{S U} & =12-N_{F}-2 N_{Q} \\
b_{0}^{S p} & =2 N_{F}+3 N_{Q}-16
\end{aligned}
$$

indicate the absence of a free magnetic phase.

\section{Appendix C. A related duality transformation}

An interesting variant on our duality transformation can be found by considering a particular relevant perturbation in the form of a nonzero superpotential. Consider a theory with symmetry group

$$
G=S O(10)_{\text {local }} \times\left[S U\left(N_{f}\right) \times S U(2)\right]_{\text {global }}
$$

and matter content

$$
\begin{aligned}
V_{\mu}^{i} & \sim(10 ; \square, 1) \\
Z_{\mu}^{X} & \sim\left(10 ; 1,2 N_{Q}-1\right) \\
Q_{I}^{A} & \sim\left(16 ; 1, N_{Q}\right) .
\end{aligned}
$$

In the absence of a superpotential, this is simply a theory of the type in (4.2) with $N_{f}+$ $2 N_{Q}-1$ vectors. However, using the fact that two $N_{Q}$ representations of $S U(2)$ can be contracted symmetrically to form a $2 N_{Q}-1$ representation, we can add the superpotential $W_{\text {tree }}=Z^{X} Q_{I} Q_{J}$ while preserving the symmetry group (2.1).

In the absence of the electric superpotential, the dual representation is given by an $S U\left(N_{f}+4 N_{Q}-8\right) \times S p\left(2 N_{Q}-2\right)$ gauge theory. The addition of the superpotential can be analyzed using the details of the duality given in (4.6)-(4.9). After performing a long but straightforward analysis and renaming various fields, one finds a magnetic theory with symmetry group

$$
\widetilde{G}=\left[S U\left(N_{f}+2 N_{Q}-7\right) \times S p\left(2 N_{Q}-2\right)\right]_{\text {local }} \times\left[S U\left(N_{f}\right) \times S U(2)\right]_{\text {global }}
$$

and matter fields

$$
\begin{aligned}
\hat{q}_{i}^{\alpha} & \sim(\square, 1 ; \bar{\square}, 1) \\
\hat{q}_{I}^{\alpha \dot{\alpha}} & \sim(\square, \square ; 1,2) \\
\hat{\hat{q}}_{\alpha\left(I_{1} \cdots I_{2 N_{Q}-2}\right)} & \sim\left(\bar{\square}, 1 ; 1,2 N_{Q}-1\right) \\
\hat{s}_{\alpha \beta} & \sim(\bar{\square}, 1 ; 1,1 ;) \\
\hat{t}_{\left(I_{1} \cdots I_{2} N_{Q}-3\right)}^{\dot{\alpha}} & \sim\left(1, \square ; 1,2 N_{Q}\right) . \\
\hat{m}^{(i j)} & \sim(1,1 ; \square, 1) \\
\hat{n}_{\left(I_{1} \cdots I_{2} N_{Q}-2\right)}^{i} & \sim\left(1,1 ; \square, 2 N_{Q}-1\right) .
\end{aligned}
$$


The superpotential has the same form as (4.9) with the obvious replacements of fields. Although this theory looks deceptively similar to the magnetic theory of Section 4, it is different in important ways. First, the field $\hat{t}$ transforms in the $2 N_{Q}$ dimensional representation of $S U(2)$, unlike the field $t$ in (4.7) which is in the $2 N_{Q}-2$. Furthermore, the "window-frame" invariant $K=Q^{4}$ is zero because of the superpotential. Instead the operator $\hat{t}^{2}$ is mapped to $Z^{2}$. Also, while the operator $m^{i j}$ is again the image of $V^{i} V^{j}$, the operator $n_{X}^{i}$ is now mapped to $V^{i} Z_{X}$.

\section{Appendix D. Renormalization group check}

In this appendix, we present the details of the two sets of duality transitions summarized in (4.13)-(4.14). At each stage, we assume that the gauge groups not involved in the duality transition are weakly coupled at the energy scale of the transition, so they can be treated as spectators. This assumption can always be satisfied for appropriate choices of the high energy coupling constants.

At ultrahigh energies, the theory has nonabelian symmetries

$G=\left[S O\left(2 N_{Q}+N_{f}-3\right) \times S U\left(N_{f}+2 N_{Q}-7\right) \times S p\left(2 N_{Q}-2\right)\right]_{\text {local }} \times\left[S U\left(N_{f}\right) \times S U(2)\right]_{\text {global }}$

and matter fields

$$
\begin{aligned}
q & \sim(1, \square, 1 ; \bar{\square}, 1) \\
q^{\prime} & \sim(1, \square, \square ; 1,2) \\
\bar{q} & \sim\left(\bar{\square}, 1 ; 1,2 N_{Q}-1\right) \\
u & \sim(\square, \bar{\square}, 1 ; 1,1) \\
t & \sim\left(1,1, \square ; 1,2 N_{Q}-2\right) \\
m & \sim(1,1,1 ; \square, 1 ;) \\
n & \sim\left(1,1,1 ; \square, 2 N_{Q}-1\right) .
\end{aligned}
$$

These fields are the same as those in (4.7) except for the absence of the field $s$ and the presence of a field $u$. We will soon identify $s$ as the bilinear $u$. The superpotential for the theory is consistent with this identification:

$$
W=m q u u q+q^{\prime} u u q^{\prime}+n q \bar{q}+q^{\prime} \bar{q} t
$$

Consider the renormalization group flow of (4.13), for which the $S O$ factor becomes strongly coupled first. The $S O\left(N_{f}+2 N_{Q}-3\right)$ gauge group contains $N_{f}+2 N_{Q}-7$ vector 
representations and no other charged matter. Such a theory is known to confine and to have an effective description in terms of a bilinear composite field uu [20]. No dynamical superpotential is generated. 11 The field $u$, if identified with $s$, leaves the remaining $S U \times S p$ theory with the gauge group, matter content, and superpotential of the theory (4.6)-(4.9). The low energy dynamics is thus equivalent to $S O(10)$ with $N_{f}$ vectors, $N_{Q}$ spinors and vanishing superpotential.

Now let us consider the more intricate behavior of (4.14) which follows from allowing the gauge coupling of the $S U$ group to grow strong first. Since the $S U$ group has only fundamentals and antifundamentals, we may take its dual using Seiberg's transformation [1]. This leads to a low energy $S U\left(2 N_{Q}+3\right)$ gauge group. The bilinears $A=q^{\prime} u, B=q^{\prime} q$, $C=q u$ and $D=q \bar{q}$ all become singlet fields in the low energy theory, while dual quarks $\hat{q}, \hat{q^{\prime}}, \hat{u}, \hat{\bar{q}}$ now appear. The field $B$ is a reducible representation under the $S U(2)$ flavor group; for reasons which will become clear we name its two irreducible components $\bar{t}$ and $\hat{t}$. Under the symmetries of the theory

$$
G=\left[S O\left(2 N_{Q}+N_{f}-3\right) \times S U\left(2 N_{Q}+3\right) \times S p\left(2 N_{Q}-2\right)\right]_{\text {local }} \times\left[S U\left(N_{f}\right) \times S U(2)\right]_{\text {global }},
$$

the matter fields transform as

$$
\begin{array}{rlrl}
A \sim(\square, 1, \square ; 1,2) & \hat{q} \sim(1, \square, 1 ; \square, 1) \\
\bar{t} \sim\left(1,1, \square ; 1,2 N_{Q}-2\right) & \hat{q}^{\prime} \sim(1, \square, \square, 1,2) \\
\hat{t} \sim\left(1,1, \square ; 1,2 N_{Q}\right) & \overline{\hat{q}} \sim\left(1, \bar{\square}, 1 ; 1,2 N_{Q}-1\right) \\
C \sim(\square, 1,1 ; \bar{\square}, 1) & \hat{u} \sim(\square, \bar{\square}, 1 ; 1,1) \\
D \sim\left(1,1,1 ; \bar{\square}, 2 N_{Q}-1\right) & t \sim\left(1,1, \square ; 1,2 N_{Q}-2\right) \\
& m \sim(1,1,1 ; \square, 1) \\
& n & \sim\left(1,1,1 ; \square, 2 N_{Q}-1\right) .
\end{array}
$$

The superpotential of this theory is

$$
W=A^{2}+\bar{t} t+A \hat{q}^{\prime} \hat{u}+\bar{t} \hat{q}^{\prime} \overline{\hat{q}}+\hat{t} \hat{q}^{\prime} \overline{\hat{q}}+C \hat{q} \hat{u}+D \hat{q} \overline{\hat{q}}+m C C+n D
$$

The fields $A, \bar{t}, t, n, D$ are massive and can be integrated out. This leaves the superpotential

$$
W=\hat{q}^{\prime} \hat{u} \hat{u} \hat{q}^{\prime}+\hat{t} \hat{q}^{\prime} \hat{\hat{q}}+C \hat{q} \hat{u}+m C C
$$

11 The theory actually has a second branch with a destabilizing dynamical superpotential. We disregard this other branch as no supersymmetric vacua are associated with it. 
The $S O\left(N_{f}+2 N_{Q}-3\right)$ group can now become strongly coupled. It has only vector representations $C$ and $\hat{u}$, so its strong dynamics can be described using Seiberg's duality [1,20]. The low energy group, independent of $N_{f}$ and $N_{Q}$, is $S O(10)$. The fields $C$ and $\hat{u}$ combine to give $S O(10)$ singlets $E=C C, F=C \hat{u}$, and $\hat{s}=\hat{u} \hat{u}$, and there are dual quarks $V$ and $v$ which are vectors of $S O(10)$. The symmetry group is now

$$
G=\left[S O(10) \times S U\left(2 N_{Q}+3\right) \times S p\left(2 N_{Q}-2\right)\right]_{\text {local }} \times\left[S U\left(N_{f}\right) \times S U(2)\right]_{\text {global }},
$$

under which the matter fields transform as

$$
\begin{array}{rlrl}
m \sim(1,1,1 ; \square, 1) & V & \sim(\square, 1,1 ; \square, 1) \\
E \sim(1,1,1 ; \bar{\square}, 1) & & \sim(\square, \square, 1 ; 1,1) \\
F \sim(1, \bar{\square}, 1 ; \bar{\square}, 1) & \hat{q}^{\prime} \sim(1, \square, \square, 1,2) \\
\hat{q} \sim(1, \square, 1 ; \square, 1) & \overline{\hat{q}} \sim\left(1, \bar{\square}, 1 ; 1,2 N_{Q}-1\right) \\
\hat{s} & \sim(1, \bar{\square}, 1 ; 1,1) \\
& \hat{t} \sim\left(1,1, \square ; 1,2 N_{Q}\right) .
\end{array}
$$

The superpotential is

$$
W=\hat{q}^{\prime} \hat{s} \hat{q}^{\prime}+\hat{t} \hat{q}^{\prime} \overline{\hat{q}}+\hat{s} v v+F \hat{q}+m E+E V V+F V v .
$$

The fields $m, E, F, \hat{q}$ are massive, and when they are integrated out the last four terms in the above superpotential are removed.

The $S U \times S p$ subgroup of this last theory has charged matter which is similar to the magnetic theory discussed in Appendix C. However, there are several minor differences. First, the field $\hat{q}$ in (C.4) is replaced by the field $v$ above. The fact that $v$ is charged under $S O(10)$ will not matter as long as $S O(10)$ is weakly coupled. Second, the superpotential (D.10) contains the extra term $\hat{s} v v$; this causes no difficulties since it is mapped under duality to a corresponding term in the electric superpotential. Third, the mesons $\hat{m}$ and $\hat{n}$ in (C.4) are missing, along with their superpotential couplings. However, it is easy to convert the duality of Appendix $\mathrm{C}$ to this situation by adding singlets $\overline{\hat{m}}$ and $\overline{\hat{n}}$ to both sides, along with the superpotential couplings $\Delta W=\overline{\hat{m}} \hat{m}+\overline{\hat{n}} \hat{n}$ on the $S U \times S p$ side and $\Delta W=\overline{\hat{m}} V V+\overline{\hat{n}} V Z$ on the $S O(10)$ side.

Thus, under the duality of Appendix C, the $S O(10)$ factor is a spectator, while the $S U \times S p$ subgroup is transformed into a second $S O(10)$ factor with $N_{Q}$ spinors, along with 
$2 N_{Q}-1$ vectors $Z^{i}$ and 10 fields $\mathcal{V}$ in the vector representation of both $S O(10)$ groups. The fields $\mathcal{M}=\hat{s} v v$ and $\mathcal{N}=v \overline{\hat{q}}$ are also present. Note that $\mathcal{M}$ decomposes under the first $S O(10)$ into a traceless symmetric tensor $\mathcal{M}_{s}$ and a singlet $\mathcal{M}_{0}$. The symmetry group of the theory is

$$
G=[S O(10) \times S O(10)]_{\text {local }} \times\left[S U\left(N_{f}\right) \times S U(2)\right]_{\text {global }},
$$

and the matter content is

$$
\begin{array}{llrl}
V & \sim(\square, 1 ; \square, 1) & \mathcal{M}_{s} & \sim(\square, 1 ; 1,1) \\
\mathcal{V} & \sim(\square, \square ; 1,1) & \mathcal{M}_{0} & \sim(1,1 ; 1,1) \\
Z & \sim\left(1, \square ; 1,2 N_{Q}-1\right) & \mathcal{N} & \sim\left(\square, 1 ; 1,2 N_{Q}-1\right) . \\
Q & \sim\left(1,16 ; 1, N_{Q}\right) & &
\end{array}
$$

The superpotential of the theory is

$$
W=Z Q Q+\mathcal{M}_{0}+\left(\mathcal{M}_{s}+\mathcal{M}_{0}\right) \mathcal{V} \mathcal{V}+\mathcal{N} \mathcal{V} Z
$$

The linear term $\mathcal{M}_{0}$ is the image under duality of the extra term $\hat{s} v v$ in the superpotential (D.10).

Because of the linear term, the equation of motion for $\mathcal{M}_{0}$ causes $\operatorname{tr} \mathcal{V} \mathcal{V}$ to condense. D-term conditions force the field $\mathcal{V}$ to develop a diagonal expectation value which breaks $S O(10) \times S O(10)$ to the diagonal $S O(10)$ subgroup. The $\mathcal{N} \mathcal{V} Z$ term gives mass to $Z$ and $\mathcal{N}$, while the $\mathcal{M V} \mathcal{V}$ term gives mass to $\mathcal{M}_{s}, \mathcal{M}_{0}$ and the 55 components of $\mathcal{V}$ which are not eaten by gauge bosons. This eliminates the entire superpotential. The remaining fields are $N_{f}$ vectors $V$ and $N_{Q}$ spinors $Q$ under the $S O(10)$ gauge group. This is the endpoint of the flow (4.14), and it matches with the endpoint of (4.13). 


\section{Figure Captions}

Fig. 1. Renormalization group flows along various flat directions of the $S O(10)$ model with $N_{f}$ vectors and $N_{Q}$ spinors. Non-singlet matter contents for each sub-theory are listed. The flat direction which connects the $S O(7)$ model with $N_{f}-3$ vectors and $2 N_{Q}$ spinors to the $G_{2}$ theory is not displayed for clarity's sake. 


\section{References}

[1] N. Seiberg, Nucl. Phys. B435 (1995) 129.

[2] P. Pouliot, Phys. Lett. B359 (1995) 108.

[3] P. Pouliot and M. Strassler, Phys. Lett. B375 (1996) 175.

[4] K. Intriligator and S. Thomas, Nucl. Phys. B473 (1996) 121; hep-th 9608046.

[5] P. Pouliot, Phys. Lett. B367 (1996) 151.

[6] E. Poppitz, Y. Shadmi and S. Trivedi, Nucl. Phys. B480 (1996) 125; Phys. Lett. B388 (1996) 561.

[7] C. Csaki, L. Randall and W. Skiba, Nucl. Phys. B479 (1996) 65; C. Csaki, L. Randall, W. Skiba and R.G. Leigh, Phys. Lett. B387 (1996) 791; L. Randall, hep-ph 9612426;

W. Skiba, hep-th 9703159; R.G. Leigh, L. Randall and R. Rattazzi, hep-ph/9704246.

[8] T. Hotta, K.-I. Izawa and T. Yanagida, Phys. Rev. D55 (1997) 415.

[9] C.-L. Chou, Phys. Lett. B391 (1997) 329.

[10] M. Bershadsky, A. Johansen, T. Pantev, V. Sadov and C. Vafa, hep-th 9612052.

[11] C. Vafa and B. Zweibach, hep-th 9701015.

[12] H. Ooguri and C. Vafa, hep-th 9702180.

[13] S. Elitzur, A. Giveon and D. Kutasov, hep-th 9702014.

[14] S. Elitzur, A. Giveon, D. Kutasov, E. Rabinovici and A. Schwimmer, hep-th 9704104.

[15] A. Brandhuber, J. Sonnenschein, S. Theisen and S. Yankielowicz, hep-th 9704044.

[16] N. Evans, C. Johnson and A.D. Shapere, hep-th 9703210.

[17] J.L.F. Barbon, hep-th 9703051.

[18] J.H. Brodie and A. Hanany, hep-th 9704043.

[19] O. Aharony and A. Hanany, hep-th 9704170.

[20] K. Intriligator and N. Seiberg, Nucl. Phys. B444 (1995) 125.

[21] M. Berkooz, Nucl. Phys. B452 (1995) 513.

[22] M. Luty, M. Schmaltz and J. Terning, Phys. Rev. D54 (1996) 7815.

[23] T. Sakai, hep-th 9701155.

[24] K. Intriligator, R.G. Leigh and M.J. Strassler, Nucl. Phys. B456 (1995) 567.

[25] J. Brodie, Nucl. Phys. B478 (1996) 123; J.H. Brodie and M.J. Strassler, hep-th 9611197 .

[26] D. Kutasov, Phys. Lett. B351 (1995) 230.

[27] P. Pouliot and M. Strassler, Phys. Lett. B370 (1996) 76.

[28] T. Kawano, Prog. Theor. Phys. 95 (1996) 963.

[29] J. Distler and A. Karch, hep-th 9611088.

[30] P. Cho, hep-th 9702059.

[31] M.A. Shifman and A.I. Vainshtein, Nucl. Phys. B359 (1991) 571.

[32] A.G. Elashvili, Funk. Anal. Pril. 6 (1972) 51.

[33] P. Cho, hep-th 9701020. 
[34] J. Harvey, D.B. Reiss and P. Ramond, Nucl. Phys. B199 (1982) 223.

[35] N. Seiberg, Phys. Rev. D49 (1994) 6857.

[36] C. Csáki, M. Schmaltz and W. Skiba, hep-th 9612207.

[37] D. Kutasov and A. Schwimmer, Phys. Lett. B354 (1995) 315.

[38] K. Intriligator, Nucl. Phys. B448 (1995) 187.

[39] R.G. Leigh and M.J. Strassler, Nucl. Phys. B447 (1995) 95.

[40] D. Kutasov, A. Schwimmer and N. Seiberg, Nucl. Phys. B459 (1996) 455.

[41] E. Witten, Phys. Lett. 117B (1982) 324.

[42] I. Affleck, M. Dine and N. Seiberg, Phys. Rev. Lett. 51 (1983) 1026.

[43] R.G. Leigh and M.J. Strassler, hep-th 9611020.

[44] A.D. Kennedy, J. Math. Phys. 22 (1981) 1330.

[45] I. Affleck, M. Dine and N. Seiberg, Phys. Lett. 140B (1984) 59.

[46] K. Intriligator and P. Pouliot, Phys. Lett. B353 (1995) 471.

[47] M.J. Strassler, in preparation.

[48] N. Maekawa and J. Sato, Prog. Theory. Phys. 96 (1996) 979.

[49] J. Wess, Lecture Notes in Physics 77, (Springer), 1978.

[50] P. West, Introduction to Supersymmetry and Supergravity, 2nd ed., (World Scientific), 1990.

[51] P. Wess and J. Bagger, Supersymmetry and Supergravity, (Princeton University Press) 1992.

[52] M. Berkooz, Nucl. Phys. B466 (1996), 75. 


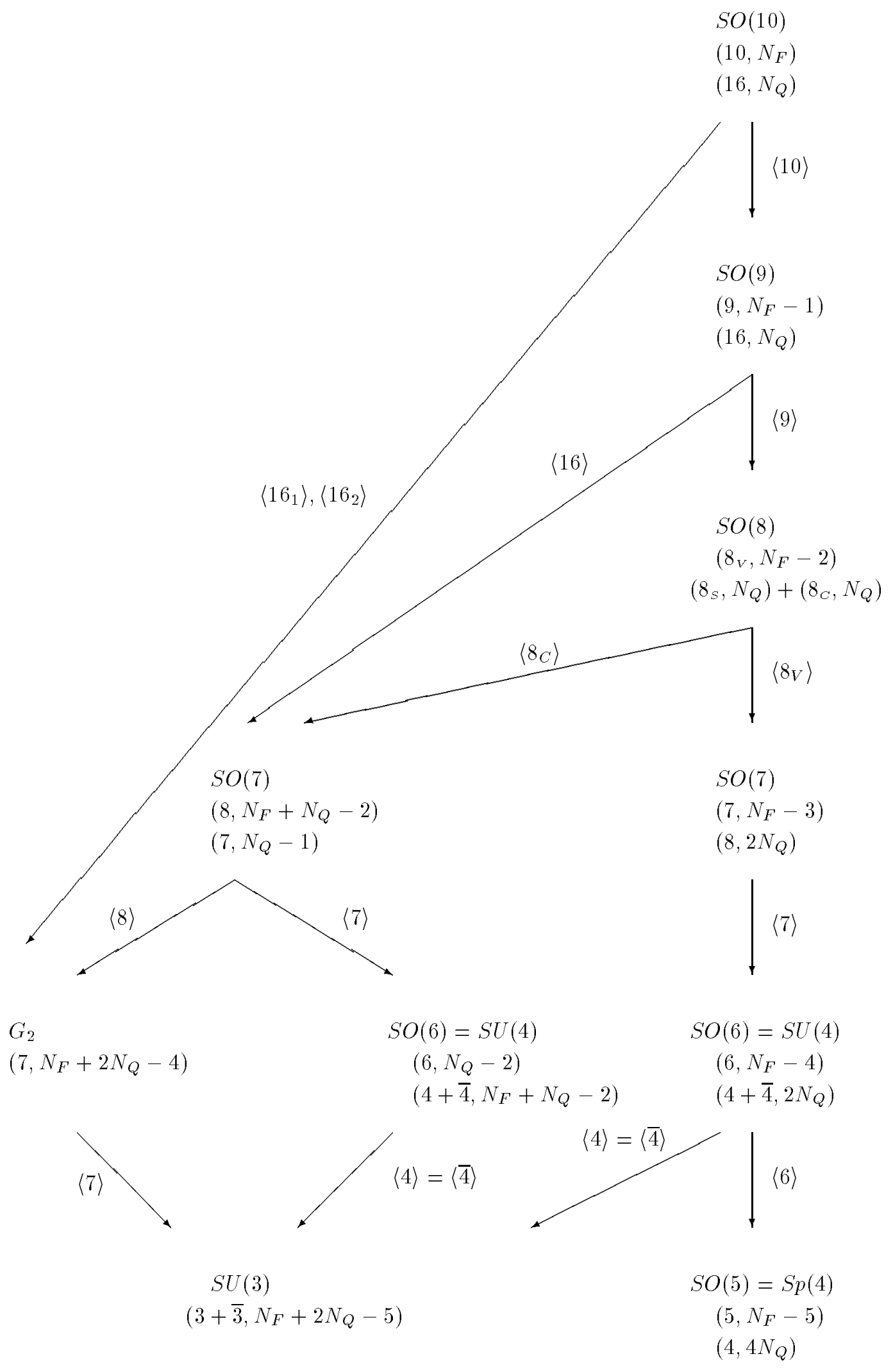

Figure 1 